\title{
G somatem \\ Functional Role of the SLC7A11-AS1/XCT Axis in the Development of Gastric Cancer Cisplatin-resistance by a GSH-dependent Mechanism
}

\section{Yajun Luo}

The First Affiliated Hospital of Chengdu Medical College: Chengdu Medical College The First Affiliated Hospital

\section{Wanping Xiang}

Affiliated Hospital of North Sichuan Medical College

\section{Zilin Liu}

The First Affiliated Hospital of Chengdu Medical College: Chengdu Medical College The First Affiliated Hospital

\section{Lin Yao}

The First Affiliated Hospital of Chengdu Medical College: Chengdu Medical College The First Affiliated Hospital

\section{Linghan Tang}

The First Affiliated Hospital of Chengdu Medical College: Chengdu Medical College The First Affiliated Hospital

\section{Pengcheng Ye}

Affiliated Hospital of North Sichuan Medical College

\section{Jingyu Deng}

Tianjin Medical University Cancer Institute and Hospital: Tianjin Tumor Hospital

\section{Jiangwei Xiao ( $\nabla$ xiaojiangwei2018@163.com )}

The First Affiliated Hospital of Chengdu Medical College: Chengdu Medical College The First Affiliated Hospital

\section{Research}

Keywords: Long non-coding RNA, Gastric cancer, SLC7A11-AS1, xCT, Resistance

Posted Date: May 7th, 2021

DOI: https://doi.org/10.21203/rs.3.rs-406690/v1

License: (9) This work is licensed under a Creative Commons Attribution 4.0 International License. Read Full License 
Version of Record: A version of this preprint was published at Free Radical Biology and Medicine on March 1st, 2022. See the published version at https://doi.org/10.1016/j.freeradbiomed.2022.03.026. 


\section{Abstract}

Background: Resistance to platinum-based chemotherapy is a major obstacle in gastric cancer (GC) treatment. Abundant long noncoding RNAs (IncRNAs) are reported to play important roles in tumorigenesis and drug resistance biology. We aimed to investigate the roles and mechanisms of SLC7A11-AS1 in GC cisplatin resistance.

Methods: Quantitative real-time PCR (qRT-PCR), RNA fluorescence in situ hybridization (RNA-FISH), immunofluorescence staining and immunohistochemistry were performed on GC tissues or cells to assess expression of SLC7A11-AS1/xCT axis. Cell migration, invasion and apoptosis ability were evaluated by wound healing, transwell and flow cytometry assays. The functional roles of SLC7A11AS1/xCT axis in GC cisplatin resistance were demonstrated by a series of in-vitro and in-vivo experiments.

Results: We report that the SLC7A11-AS1 and $x$ CT are involved in cisplatin resistance in GC. SLC7A11AS1 was downregulated and XCT was upregulated in cisplatin-resistant GC tissues and cell lines. GC patients with low expression of SLC7A11-AS1 and high expression of XCT had a poor prognosis and relatively poor response to chemotherapy. Overexpression of SLC7A11-AS1 weakened GC growth, reduced intracellular GSH biosynthesis, enhanced intracellular reactive oxygen species (ROS) and conferred sensitivity to cisplatin to resistant GC cells in vitro and in vivo. Mechanistically, SLC7A11-AS1 directly suppressed $x C T$ expression, while miR-33a-5p remarkably reduced SLC7A11-AS1 and xCT expression by directly targeting the SLC7A11-AS1 and xCT 3'UTRs. In addition, we found that low SLC7A11-AS1 expression activated the p38MAPK-JNK signaling pathway, and increased the expression of cisplatin export gene ATP7A and the GSH biosynthesis gene GCLM in GC.

Conclusions: These findings suggest that the SLC7A11-AS1/xCT axis is a crucial therapeutic target to overcome platinum-related resistance for GC treatment.

\section{Background}

Gastric cancer (GC) is the fifth most common malignancy and third leading cause of cancer-related death worldwide [1]. Over one million new diagnoses and an estimated 783,000 deaths occur every year worldwide, while more than $40 \%$ of the new GC diagnoses and deaths occur in China [2,3]. Radical gastrectomy combined with postoperative adjuvant chemotherapy is the main curative treatment for GC [4]. The 5-year overall survival (OS) rate of patients treated with adequate complete surgical resection of locally advanced GC and adjuvant chemotherapy has generally improved by $10-15 \%[5,6]$. At present, neoadjuvant chemotherapy for advanced GC is also an the important therapeutic measure to improve radical resection and the five-year survival rate of GC patients. Although traditional treatments bring survival benefits to $\mathrm{GC}$ patients, many patients experience recurrence soon after postoperative chemotherapy or fail to achieve good results with neoadjuvant chemotherapy, indicating that tumor heterogeneity facilitates escape from cytotoxic chemotherapy. As with other types of cancer, GC is 
heterogeneous, GC drug resistance is a complicated process involving diverse factors and molecules, and the mechanisms underlying the drug resistance process are still poorly understood.

Platinum-based chemotherapy, such as cisplatin, is a widely used treatment across a wide spectrum of malignancies including GC $[7,8]$. However, despite an initial response to cisplatin, a large number of cancer patients eventually relapse and develop drug resistance [9]. Cisplatin exerts the most prominent antineoplastic effects that have been fully ascribed to generating unrepairable DNA lesions and inducing mitochondrial apoptosis [10]. While multiple cisplatin resistances mechanisms have been proposed and classified based on functional and hierarchical parameters, such as pretarget resistance, on-target resistance, posttarget resistance, and off-target resistance [10], the most prominent mechanism of resistance appears to be increasing drug efflux and reducing influx or sequestration to lower cisplatin concentrations within cells [11]. In this process, glutathione (GSH) is a major antioxidant involved in cisplatin metabolism in cancer cells, which protects cells against reactive oxygen species (ROS) induced by the cytotoxic effects of cisplatin [12]. Cysteine is a rate limiting precursor in intracellular GSH synthesis [13], which is imported into cells by the system $x^{-}{ }^{-}$cystine-glutamate exchange transporter [14]. System $\mathrm{xc}^{-}$is composed of a heavy-chain subunit (SLC3A2) and a light-chain subunit (SLC7A11, also known as $\mathrm{XCT}$ ). $\mathrm{XCT}$ expression on the cell surface is essential for the uptake of cystine required for intracellular GSH synthesis and is a vital determinant of the intracellular redox balance. Accumulating evidence now suggests that upregulation of XCT is a mechanism of multidrug resistance in colorectal cancer [15], bladder cancer [16] and gastric cancer [17]. Therefore, targeting XCT appears to be a promising strategy to overcome cancer resistance to chemotherapy. However, the effects of targeting $\mathrm{xCT}$ to reverse drug resistance have not been well clarified.

Notably, multiple lines of evidence have recently highlighted the role of noncoding RNAs in the development and maintenance of the GC drug-resistant phenotype [18, 19], especially miRNAs and IncRNAs, which play important roles in regulating chemotherapy sensitivity in GC. For instance, the IncRNA HOTAIR can reverse DDP resistance in GC cells through suppression of PI3K/Akt signaling by increasing miR-34a [20], and the IncRNA MALAT1 induces GC cell resistance to cisplatin through miR23b-3p promoting autophagy [21]. SLC7A11-AS1 is an overlapping cis-natural antisense transcript at the xCT gene locus. In a previous study, we demonstrated that SLC7A11-AS1 is downregulated in GC and functions as a tumor suppressor gene to inhibit cell growth by suppressing XCT [22]. Interestingly, another study showed that SLC7A11-AS1 promotes chemoresistance by reducing intracellular ROS via stabilization of nuclear factor erythroid-2-related factor 2 (NRF2) in pancreatic cancer [23]. The molecular mechanisms involving SLC7A11-AS1 in drug resistance are not clear and remain controversial. Therefore, this study investigated whether the SLC7A11-AS1/xCT axis can overcome GC cell resistance to cisplatin treatment.

In our current study, we presented evidence that SLC7A11-AS1 was downregulated in cisplatin-resistant GC cells and involved in drug resistance. SLC7A11-AS1 was found to interact with miR-33a-5p to contribute to cisplatin resistance in GC by modulating the expression of XCT in vitro and in vivo and, as a result, increasing the levels of intracellular GSH to maintain the intracellular redox balance after cisplatin 
treatment. Here, we demonstrate a potential clinical approach to reverse platinum-related resistance and suggest that the biochemical consequences of this mechanism of resistance may be used to stratify chemotherapeutic drug choice for patients with advanced cancer.

\section{Materials And Methods}

\section{Patients and tumor tissues}

A total of 140 human GC patients were enrolled in this study, and 24 GC patients were tested for multidrug resistance by primary culture of GC tissues. All tissues were obtained at the time of surgery between 2016 and 2018 at the First Affiliated Hospital of Chengdu Medical College (Sichuan, China), and detailed clinicopathological parameters were collected. Following excision, all tissues were immediately frozen in liquid nitrogen and stably stored at $-80^{\circ} \mathrm{C}$ until use. All patients gave written informed consent under protocols approved by the institutional review boards at the First Affiliated Hospital of Chengdu Medical College, Chengdu, China.

\section{Cell lines, cell cultures and reagents}

The primary human GC cell lines HEK293T and SGC7901 were purchased from the Cell Bank of the Chinese Academy of Sciences (Shanghai, China) and grown in DMEM (Gibco BRL, Gaithersburg, MD, USA) supplemented with 10\% fetal bovine serum (Gibco BRL, Gaithersburg, MD, USA) and antibiotics (100 IU/ml penicillin and $100 \mu \mathrm{g} / \mathrm{ml}$ streptomycin). The cisplatin-resistant cell subline SGC7901/DDP was generated from the sensitive parental GC cell line SGC7901 in our laboratory. Cells were grown in increasing cisplatin concentrations (the final concentration of DDP was $1 \mu \mathrm{g} / \mathrm{ml}$ ) for 4 months. Cisplatin (DDP; Sigma, Inc., St. Louis, MO, USA; $1 \mu \mathrm{g} / \mathrm{ml}$ ) was added to the culture medium of appropriate cell sublines to maintain the DDP-resistant phenotype. Cisplatin and sulfasalazine were purchased from Sigma-Aldrich (St. Louis, MO, USA).

\section{Quantitative real-time polymerase chain reaction}

Total RNA was extracted from tissues using TRIzol reagent (Invitrogen, Carlsbad, CA, USA), and reverse transcription reactions were performed using the GoScript Reverse Transcription (RT) System (Promega, Madison, WI, USA) according to the manufacturer's protocol. Real-time PCR was performed using a standard SYBR Green PCR kit (Roche Diagnostics GmbH, Mannheim, BW, Germany) according to the manufacturer's instructions. GAPDH and U6 were used as the references for IncRNAs and miRNAs. Primers were synthesized by Sangon Biotech (Shanghai, China), and their sequences are listed in Table $S 1$. The $2^{-\Delta \Delta C t}$ method was used to quantify relative gene expression. Each sample was analyzed in triplicate. 


\section{Adenoviral infection of cell lines}

An SLC7A11-AS1-overexpression adenoviral vector (Adv-SLC7A11-AS1-GFP) and control adenoviral vector (Adv-GFP) were purchased from GeneChem (Shanghai, China). pHBAd-RNAi-GFP targeting SLC7A11-AS1 and the negative control pHBAd-GFP were designed and synthesized by Hanbio (Shanghai, China). The specific siRNA sequence for SLC7A11-AS1 was 5'-aggtgtctgtgagggtgtttctgta-3'. Cells were seeded at an $80 \%$ density in 6 -well plates before infection, and the culture medium was changed $8 \mathrm{~h}$ after infection. After infection for $48 \mathrm{~h}$, the infected cells were harvested for use.

\section{Wound healing and transwell assays}

Cells were cultured in 6-well plates, followed by scratching to create an artificial wound using a 200- $\mu$ l micropipette tip in the center of each well. Then, the wounded monolayers were washed with phosphatebuffered saline (PBS) to remove cell debris and incubated with serum-free medium. At four time points, the distance between the two edges of the wound was calculated at three different positions.

An invasion assay was performed using a 24-well Transwell chamber coated with Matrigel (BD Pharmingen, San Jose, CA, USA) and separated by polycarbonate membranes with 8- $\mu \mathrm{m}$ pores (Costar, Cambridge, MA, USA). Then, $2.0 \times 10^{3}$ cells were seeded in the upper chamber with serum-free medium, and the lower chamber was filled with culture medium containing $10 \%$ fetal bovine serum. Invaded cells were fixed with $4 \%$ paraformaldehyde (Sigma Aldrich, St. Louis, MO, USA), stained with $1 \%$ crystal violet (Beyotime, Shanghai, China) and quantified. A migration assay was conducted in a similar fashion without Matrigel coating. All experiments were repeated independently in triplicate.

\section{Cell apoptosis}

Cells were harvested after adenoviral infection for $48 \mathrm{~h}$, and the cell density was adjusted to $5 \times 10^{5}$. Then, the cells were washed with precooled phosphate buffer solution (PBS) 2 times and resuspended after the addition of $100 \mu \mathrm{l}$ buffer solution. According to the manufacturer's protocol for an Annexin V-APC/7-ADD apoptosis detection kit (KeyGEN BioTECH, China), $5 \mu$ Innexin V-APC and $5 \mu$ 7-amino-actinomycin D (7$A D D$ ) were added to the cultured cells at room temperature for $15 \mathrm{~min}$. The stained cells were then analyzed by using a BD FACS Canto II flow cytometer (BD, USA). The flow cytometry assay was repeated three times.

\section{RNA fluorescence in situ hybridization and immunofluorescence staining}

A Cy3-labeled SLC7A11-AS1 probe was generated by RiboBio (Guangzhou, China). Slides were fixed with $4 \%$ paraformaldehyde for $10 \mathrm{~min}$ at room temperature, followed by permeabilization at $4^{\circ} \mathrm{C}$ for $5 \mathrm{~min}$. The 
slides were hybridized overnight with the SLC7A11-AS1 probe and then washed three times with a graded saline sodium citrate solution.

For immunofluorescence staining, cells were fixed with $4 \%$ paraformaldehyde for 30 min, stained with a rabbit anti-xCT antibody (1:100 dilution; Proteintech, China) overnight, washed with PBS three times, incubated with a Cy3-goat anti-rabbit IgG $(\mathrm{H}+\mathrm{L})$ secondary antibody (1:50 dilution; Beyotime, China) and washed with PBS three times. The nuclei were counterstained with DAPI. Fluorescence images were acquired with a confocal microscope (Leica, Wetzlar, Germany).

\section{Immunohistochemistry}

Tumor tissues were fixed in $4 \%$ paraformaldehyde, embedded in paraffin, and sectioned at $5 \mu \mathrm{m}$. Briefly, paraffin sections were dewaxed in xylene and dehydrated through a graded series of alcohol solutions. The sections were then treated with protein blockers and stained with hematoxylin and eosin or incubated with an anti-xCT antibody (1:200; Abcam, Cambridge, MA, USA) according to standard protocols.

\section{Cell viability assays}

A CCK8 assay was used to analyze cisplatin toxicity. A total of 1000 cells were seeded in 96 -well plates and cultured for $48 \mathrm{~h}$. Then, a CCK8 solution was added and incubated for one hour. The absorbance at $450 \mathrm{~nm}$ was determined for each sample.

\section{Luciferase reporter assay}

Wild-type (WT) or mutant (Mut) SLC7A11-AS1 and xCT 3'UTR sequences containing the miR-33a-5p binding site were inserted into the psiCHECK-2 vector (Promega, Madison, WI, USA) to generate psiCHECK-SLC7A11-AS1-WT, psiCHECK-SLC7A11-AS1-Mut, psiCHECK-xCT-WT and psiCHECK-xCT-Mut plasmids. Then, the luciferase plasmids $(0.16 \mu \mathrm{g})$ and miR-33a-5p mimics/mimics NC (5 pmol) were transfected into HEK293T cells using Lipofectamine 2000 (Invitrogen, Carlsbad, CA, USA). Luciferase activities were quantified sequentially by the Dual-Luciferase ${ }^{\circledR}$ Reporter Assay System (Promega, Madison, WI, USA) in accordance with the manufacturer's instructions after 48 hours of transfection. The relative firefly luciferase activity was normalized to the Renilla luciferase activity.

\section{Western Blotting}

Total protein was extracted, and the concentration was determined using a BCA protein assay kit (Beyotime, China). Protein samples were electrophoresed by SDS-PAGE and transferred to a PVDF membrane. The membrane was incubated with primary antibodies against $x C T, A S K 1, p-P 38, P 38, p-J N K$, JNK and GAPDH (Abcam, Cambridge, MA, USA) according to the manufacturer's instructions. 


\section{Cellular glutathione and ROS production detection}

Cellular glutathione levels were measured by a GSH-Glo ${ }^{\text {TM }}$ glutathione assay kit (Promega, Madison, WI,

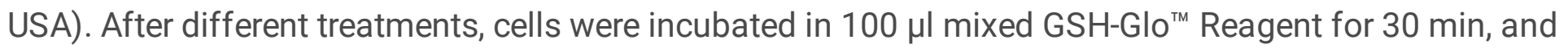
then $100 \mu$ reconstituted Luciferin Detection Reagent was added for 15 min. Luminescence signals were detected using a Fluoroskan luminescence scanner (Thermo Fisher, Rockford, IL, USA).

Cellular ROS levels were measured by the Reactive Oxygen Species Assay Kit (Beyotime, China). Cells were incubated with $10 \mu \mathrm{M}$ 2',7'-dichlorodihydrofluorescein diacetate (DCFH-DA) and the supernatants of differently treated cell lysates for $30 \mathrm{~min}$ at room temperature. The fluorescence intensity of the cells was analyzed by FCM (BD FACSCalibur; BD Biosciences).

\section{Mouse xenograft study}

BALB/c-nude mice (4 weeks old) were randomly divided into three groups ( $\mathrm{n}=6$ per group). SGC7901/DDP cells $\left(5 \times 10^{6}\right)$ infected with OE-SLC7A11-AS1, OE-NC or a control vector were subcutaneously implanted in the right flank of BALB/c-nude mice. For a chemoresistance study, sulfasalazine $(500 \mathrm{mg} / \mathrm{kg})$ was administered every 3 days or cisplatin $(2 \mathrm{mg} / \mathrm{kg})$ was administered every 5 days by intraperitoneal injection beginning when the tumor volume reached $100 \mathrm{~mm}^{3}$. Tumor volumes $\left(V=a \times b^{2} / 2\right)$ were measured every 3 days. In addition, tumor weights were determined after 6 weeks. All animal experimental procedures were approved by the Animal Care and Use Ethics Committee of the First Affiliated Hospital of Chengdu Medical College.

\section{Statistical analyses}

All statistical data were analyzed with GraphPad Prism 7.0 (GraphPad Software, La Jolla, CA). Differences between groups were analyzed using Student's t-test or two-way ANOVA. Data are presented as the mean \pm standard deviation (SD). All the tests were two-tailed, and $\mathrm{P}<0.05$ was considered statistically significant.

\section{Results}

\section{SLC7A11-AS1/xCT axis expression and prognosis}

In a previous study, we identified that SLC7A11-AS1 is an overlapping cis-natural antisense transcript at the $\mathrm{xCT}$ gene locus and that SLC7A11-AS1 is downregulated and $\mathrm{xCT}$ is upregulated in GC patients. Then, we further determined the SLC7A11-AS1 and xCT expression levels in tumor and paired adjacent nontumor tissues obtained from 140 patients with GC. qRT-PCR analysis revealed significantly lower SLC7A11-AS1 expression and higher xCT expression in GC tissues than in paired adjacent nontumor tissues (Fig. 1A). To further confirm the differential expression of SLC7A11-AS1 and XCT between GC and 
paired adjacent nontumor tissues, we used fluorescence in situ hybridization (FISH) to visualize SLC7A11-AS1 expression and immunohistochemistry to visualize XCT expression in tumor and paired nontumor tissues (Fig. 1B). The results showed that the expression level of SLC7A11-AS1 in human GC tissues was lower than that in normal tissues, while the expression level of XCT in normal tissues was higher than that in GC tissues.

To analyze the associations between SLC7A11-AS1 and xCT expression and the prognosis of patients with GC, we used Kaplan-Meier analysis and the log-rank test (http://kmplot.com/analysis/index.php) to evaluate the effects of SLC7A11-AS1 and XCT expression and clinicopathological characteristics on overall survival (OS), first progression (FP) and post-progression survival (PPS). Interestingly, we found that low SLC7A11-AS1 expression in tumors was associated with poor PPS ( $P=0.022$; Fig. 1C), whereas no significant associations between SLC7A11-AS1 expression and OS $(P=0.210$; Fig. 1C) or FP $(P=$ 0.150; Fig. 1C) were observed. Similarly, we found that high $\mathrm{xCT}$ expression in tumors was associated with poor OS ( $P=0.015$; Fig. 1C), FP $(P<0.001$; Fig. $1 C)$ and PPS ( $P=0.003$; Fig. $1 C)$. In a multivariate Cox regression model (Table S2), high SLC7A11-AS1 expression levels (HR, $0.61 ; 95 \% \mathrm{Cl} 0.40$ to 0.94 ) in tumors were an independent predictive factor for PPS prognosis. On the other hand, high $\mathrm{xCT}$ expression in tumors was an independent risk factor for OS (HR, 1.28; 95\% $\mathrm{Cl} 1.05$ to 1.57$)$, $\mathrm{FP}(\mathrm{HR}, 1.59 ; 95 \% \mathrm{Cl} 1.04$ to 2.04$)$ and PPS (HR, $1.61 ; 95 \% \mathrm{Cl} 1.17$ to 2.21$)$. Interestingly, the main cause of poor FP and PPS is often considered to be related to the failure of adjuvant chemotherapy after surgery. These results indicated that low expression of SLC7A11-AS1 and high expression of XCT play important roles in drug resistance in GC.

\section{SLC7A11-AS1/xCT axis as a biomarker for chemotherapy resistance in GC patients}

To examine whether SLC7A11-AS1 and xCT expression can predict the response to chemotherapy, we analyzed the associations between SLC7A11-AS1/xCT axis expression and cisplatin-resistant outcomes in 24 patients with GC by primary culture testing with five different concentrations of cisplatin. According to the resistance index, we set 250 as the cutoff point: cisplatin resistance was defined as an index greater than or equal to 250 , while cisplatin sensitivity was defined as an index less than 250 . The clinicopathological characteristics of GC patients with cisplatin resistance tests are shown in Table S3. Based on the above criteria, cisplatin resistance occurred in 11 of the $24(45.83 \%)$ patients with GC, wherein 13 of the 24 (54.17\%) patients were cisplatin sensitive. We then tested whether the SLC7A11AS1/xCT axis is functionally involved in GC cisplatin resistance. qRT-PCR analysis of molecules in this axis in cisplatin-resistant tumor tissues versus cisplatin-sensitive tumor tissues revealed that SLC7A11AS1 was expressed at low levels in the cisplatin-resistant tumor tissues, while XCT was overexpressed (Fig. 2A). Furthermore, cisplatin-resistant tumor tissues showed low SLC7A11-AS1 expression compared with cisplatin-sensitive tumor tissues by FISH (Fig. 2B), and xCT overexpression was visualized in cisplatin-resistant tumor tissues compared with cisplatin-sensitive tumor tissues by immunohistochemistry (Fig. 2C). 
To further confirm that SLC7A11-AS1/xCT axis expression is related to cisplatin resistance in GC, we created the cisplatin-resistant cell subline SGC7901/DDP from the sensitive parental GC cell line SGC790. The cisplatin half maximal inhibitory concentration $\left(\mathrm{IC}_{50}\right)$ of the cisplatin-sensitive SGC-7901 cell line was $14.02 \pm 0.215 \mu \mathrm{M}$, and the cisplatin $\mathrm{IC}_{50}$ of the cisplatin-resistant SGC-7901/DDP cell line was 20.42 $\pm 0.083 \mu \mathrm{M}$ (Fig. 2D). To confirm the relationship, qRT-PCR was performed to analyze SLC7A11-AS1/xCT axis expression in SGC7901 and SGC7901/DDP cells. We found that the SLC7A11-AS1 expression level was decreased in SGC7901/DDP cells (Fig. 2E) and the mRNA and protein levels of XCT were upregulated in SGC7901/DDP cells (Fig. 2E), which was also validated by immunofluorescence staining (Fig. 2F). Therefore, these findings strongly suggest that SLC7A11-AS1/XCT axis expression is a predictor of the response to cisplatin-based adjuvant chemotherapy.

\section{Biological functions of SLC7A11-AS1}

To evaluate the tumor-suppressive properties and effects of SLC7A11-AS1 on GC, adenoviral infectionmediated knockdown and overexpression were used to exogenously manipulate the expression of SLC7A11-AS1 in the GC cell line SGC7901. Then, Transwell invasion and migration assays showed that SLC7A11-AS1 overexpression significantly inhibited cell invasion and migration compared with control expression. In contrast, knocking down SLC7A11-AS1 expression significantly promoted cell invasion and migration (Fig. 3A). In addition, SLC7A11-AS1 overexpression attenuated the migration and invasion of SGC7901 cells, as measured by wound healing assays. In contrast, knocking down SLC7A11-AS1 in SGC7901 cells promoted the migration of these tumor cells (Fig. 3B). Flow cytometric analysis was performed to further examine whether SLC7A11-AS1 affects GC cell apoptosis. The results revealed that SLC7A11-AS1 overexpression significantly promoted cell apoptosis compared with control expression. Knocking down SLC7A11-AS1 significantly suppressed cell apoptosis (Fig. 3C). Taken together, these data indicate that SLC7A11-AS1 plays an important role in GC progression.

\section{SLC7A11-AS1 suppresses XCT expression at the transcriptional level, and miR-33a-5p suppresses SLC7A11- AS1/xCT axis expression during cisplatin resistance}

In a previous study, we identified that SLC7A11-AS1 is negatively related to xCT. To understand the mechanism underlying SLC7A11-AS1/xCT axis regulation in GC drug resistance, we developed an SLC7A11-AS1-knockdown model and SLC7A11-AS1-overexpressing model using infection with an antiSLC7A11-AS1 siRNA adenoviral vector or SLC7A11-AS1-overexpression adenoviral vector, respectively, in SGC7901 and SGC7901/DDP cells. The results indicated that knocking down SLC7A11-AS1 increased the expression levels of $x C T$, while overexpressing SLC7A11-AS1 reduced the expression levels of $x C T$ in SGC7901 and SGC7901/DDP cells (Fig. 4A). Moreover, we used immunofluorescence staining to visualize $\mathrm{xCT}$ expression in these cells, and $\mathrm{xCT}$ was upregulated or downregulated after SLC7A11-AS1 knockdown or overexpression, respectively (Fig. 4B). xCT is the sense-cognate gene for SLC7A11-AS1, 
suggesting that $x C T$ is an SLC7A11-AS1 target. Given the overlap between SLC7A11-AS1 and xCT, we hypothesized that SLC7A11-AS1 can exert its biological effects via xCT modulation. SLC7A11-AS1 was mainly expressed in the cytoplasm (Fig. 4C).

To better understand the relationship between SLC7A11-AS1 and xCT, we used DIANA and TargetScan tools to search for potential miRNAs correlated with these two genes. Through bioinformatic analysis, we found that the SLC7A11-AS1 and XCT 3'UTR sequences contain a miR-33a-5p binding site. Additionally, we found that the expression level of miR-33a-5p was upregulated in SGC7901/DDP cells compared with SGC7901 cells and was significantly upregulated after depletion of SLC7A11-AS1 in SGC7901 cells (Fig. 4D). Overexpression of miR-33a-5p through miR-33a-5p mimics depressed SLC7A11-AS1 and xCT levels in SGC7901 cells (Fig. 4E). To determine whether the SLC7A11-AS1 and XCT 3'UTRs can be direct targets of miR-33a-5p in living cells, we constructed luciferase reporter vectors using wild-type and mutant SLC7A11-AS1 and xCT 3'UTR sequences (mutation of the miR-33a-5p seed sites) (Supplementary Figure S1). According to the results of the luciferase reporter assay, miR-33a-5p mimics suppressed the activity of SLC7A11-AS1-wt and XCT-3'UTR-wt, while there were no significant changes in the activity of SLC7A11-AS1-mut or XCT-3'UTR-mut in HEK293 cells (Fig. 4E), indicating that miR-33a-5p interacts with SLC7A11-AS1 and $x C T$, and increased expression of miR-33a-5p could induce cisplatin resistance in GC cells by reducing the levels of SLC7A11-AS1 and XCT.

\section{SLC7A11-AS1 regulated the intracellular ROS and GSH by targeting the cystine/glutamate exchanger XCT}

To investigate the effects of SLC7A11-AS1 involved in regulating intracellular ROS and GSH by modulating the cystine/glutamate exchanger xCT, we detected intracellular ROS and GSH after treatment with the XCT inhibitor sulfasalazine (SSA), silencing or upregulating SLC7A11-AS1 or combining the approaches (Fig. 5A, 5B). Silencing SLC7A11-AS1 decreased the intracellular level of ROS and increased the intracellular level of GSH in SGC-7901 and SGC-7901/DDP cells. Conversely, upregulation of SLC7A11-AS1 increased the intracellular level of ROS and decreased the intracellular level of GSH. Moreover, treatment with SSA resulted in significant upregulation of intracellular ROS and a reduction in intracellular GSH in SGC-7901 and SGC-7901/DDP cells. We noticed that depletion of SLC7A11-AS1 reduced the intracellular level of SSA-induced ROS and enhanced the intracellular level of SSA-induced GSH. However, increasing the SLC7A11-AS1 level enhanced the intracellular level of SSA-induced ROS and reduced the intracellular level of SSA-induced GSH. These findings suggest that SLC7A11-AS1 reduces intracellular ROS and increases intracellular GSH by regulating XCT in GC cells.

\section{The SLC7A11-AS1/xCT axis mediated cisplatin resistance through increased production of GSH and decreased production of ROS}


To investigate how the SLC7A11-AS1/xCT axis regulates cisplatin resistance in GC cells, we compared the intracellular ROS and GSH levels in cisplatin-sensitive SGC-7901 cells and the corresponding resistant SGC7901/DDP cells. We found significantly increased concentrations of GSH and deceased concentrations of ROS in the cisplatin-resistant cells (Fig. 6A). Next, we investigated the effect of SLC7A11-AS1 and involvement of SSA involvement related to the toxicity of cisplatin (Fig. 6B). SSA significantly sensitized cisplatin-resistant SGC7901/DDP cells. Similarly, cisplatin sensitivity was restored following increased expression of SLC7A11-AS1. Furthermore, cotreatment with SSA and enhancement of the SLC7A11-AS1 level increased cisplatin sensitivity more than either single approach in cisplatinresistant SGC7901/DDP cells, indicating that the combination effect is synergistic rather than additive. These results indicate that the depletion of SLC7A11-AS1 in cisplatin-resistant GC cells may increase the expression of XCT and thus increase the production of intracellular GSH and decrease the level of intracellular ROS, which may be responsible for cisplatin resistance.

To further reveal the cisplatin resistance-related roles of the SLC7A11-AS1/xCT axis in vivo, SGC7901/DDP xenografts with SLC7A11-AS1 overexpression or control expression were established subcutaneously in BALB/c mice. Sulfasalazine $(500 \mathrm{mg} / \mathrm{kg}$ ) was administered every 3 days, or cisplatin $\left(2 \mathrm{mg} / \mathrm{kg}\right.$ ) was administered every 5 days beginning when the tumor volume reached $100 \mathrm{~mm}^{3}$. SLC7A11-AS1 overexpression significantly potentiated cisplatin cytotoxicity, as shown by the approximately $45 \%$ decrease in tumor growth and $33 \%$ decrease in tumor weight, while sulfasalazine treatment decreased tumor growth by approximately $40 \%$ and decreased tumor weight by $39 \%$ in vivo (Fig. 6C). Notably, the overexpression of SLC7A11-AS1 reversed the sensitivity of SGC7901/DDP xenografts to cisplatin compared with that of control xenografts, and the use of cisplatin exhibited excellent tumor growth suppression with no significant change in body weight (Figure S2). Moreover, the expression level of $\mathrm{XCT}$ was higher in the control group than in the OE-SLC7A11-AS1 group or sulfasalazine-treated group (Fig. 6D). These results indicate that the SLC7A11-AS1 1 low $/ x C T^{\text {high }}$ axis can protect cancer cells from the cytotoxicity of cisplatin by enhancing the biosynthesis of GSH and reducing the production of ROS.

\section{Depletion of SLC7A11-AS1 promotes cisplatin resistance via cisplatin export, GSH biosynthesis, and p38MAPK-JNK signaling activation}

To further identify changes in the expression of cisplatin metabolism-related genes, cisplatin import (CTR1) and export (ATP7A and ATP7B) and GSH biosynthesis (GCLC, GCLM, GSR and 4F2HC) were evaluated for associations with cisplatin resistance in GC cells. The expression of mRNAs important for cisplatin transport was measured using qPCR. We compared matched cisplatin-sensitive and cisplatinresistant GC cell lines and revealed that ATP7A, GCLM and GSR mRNAs were significantly upregulated in the resistant cell line SGC-7901/DDP (Fig. 7A). Then, we tested whether SLC7A11-AS1 is functionally involved with these transporters, and ATP7A, GCLC and GCLM mRNAs were significantly upregulated in 
SGC-7901/DDP cells after SLC7A11-AS1 silencing (Fig. 7A). Therefore, we further confirmed that SLC7A11-AS1 is functionally involved in GC cisplatin resistance via cisplatin export (ATP7A) and GSH biosynthesis (GCLM and $\mathrm{xCT}$ ).

To test whether the SLC7A11-AS1-mediated downregulation of XCT plays a role in p38MAPK-JNK signaling, we detected the levels of those signaling molecules and found that depletion of SLC7A11-AS1 activated p38MAPK-JNK signaling, while overexpression of SLC7A11-AS1 suppressed activated p38MAPK-JNK signaling (Fig. 7B). These data indicate that the abilities of SLC7A11-AS1 to inhibit proliferation, invasion, migration and apoptosis are largely attributable to the ability of SLC7A11-AS1 to inhibit $x C T$, which subsequently activates the p38MAPK-JNK signaling pathway. Overall, as shown in Fig. 7C, miR-33a-5p inhibits SLC7A11-AS1 and xCT expression, while SLC7A11-AS1 inhibits xCT expression. Concurrently, SLC7A11-AS1 covers the xCT 3'UTR miRNA binding site to protect xCT from degradation by miR-33a-5p, which keeps $x C T$ expression at a suitably low level to maintain ROS in the dynamic balance seen in cancer cells. The low level of ROS required for the maintenance of GC growth and chemoresistance alleviated or resisted the cytotoxicity of chemotherapeutic drugs.

\section{Discussion}

Chemotherapy is the primary treatment for advanced cancers. However, the acquisition of resistance to anticancer drugs seriously limits the effectiveness of conventional chemotherapeutics and novel biological agents and is a major cause of relapse and mortality in cancer patients. In the current study, we identified that the expression of SLC7A11-AS1 was downregulated in cisplatin-resistant GC tissues and cells and inhibited the invasion, metastasis and proliferation of GC cells. SLC7A11-AS1 dynamically inhibited $x C T$ expression by interacting with miR-33a-5p and subsequently maintained a low level of intracellular ROS by controlling the synthesis of intracellular GSH, which can neutralize the increase in ROS under oxidative stress caused by chemotherapeutic drugs that is necessary to maintain the growth and chemotherapy resistance of GC.

SLC7A11-AS1 is an overlapping cis-natural antisense transcript of XCT and may function as a doubleedged sword: a tumor suppressor or an oncogene. Its roles in the occurrence and development of tumors are controversial. In pancreatic cancer, SLC7A11-AS1 is upregulated in cancer tissues compared with adjacent nontumor counterparts and promotes cancer cell stemness and chemoresistance, working as an oncogene [23]. Similar to its role in pancreatic cancer, SLC7A11-AS1 is an oncogene that is upregulated in lung cancer tissues compared with adjacent nontumor counterparts and enhances cell proliferation, migration and invasion in lung cancer [24]. Similarly, high expression of SLC7A11-AS1 exerts as an oncogenic function and is involved in the EMT process and metastasis in hepatocellular carcinoma [25]. In contrast, SLC7A11-AS1 was indicated to be abnormally downregulated in patients with epithelial ovarian cancer and suppressed cancer cell migration and invasion or induced cancer cell apoptosis, indicating tumor suppressor functions [26]. In our previous study, we reported that SLC7A11-AS1 was downregulated in patients with GC and inhibited tumor cell growth [22]. We further used a larger sample size to prove the low expression of SLC7A11-AS1 in GC patients, indicating that SLC7A11-AS1 is a tumor 
suppressor in GC. Furthermore, overexpression of SLC7A11-AS1 inhibited cell invasion, migration and cisplatin resistance and induced apoptosis, while depletion of SLC7A11-AS1 promoted cell invasion, migration and cisplatin resistance and inhibited apoptosis. In this study, we used a large amount of real and effective data to fully prove the protumor effect of low expression or deletion of SLC7A11-AS1 in the occurrence and development of GC. Low expression of SLC7A11-AS1 was associated with shorter PPS, further supporting the tumor suppressor role of SLC7A11-AS1 in GC. Therefore, SLC7A11-AS1 plays a dual role in cancers, and its function depends on the pathological type of cancer or the tumor microenvironment.

Accumulating evidence has demonstrated that SLC7A11-AS1 and $\mathrm{xCT}$ are responsible for resistance to anticancer agents $[11,23,27]$. Yang et al. reported that SLC7A11-AS1 promoted gemcitabine chemoresistance by reducing intracellular ROS by stabilizing Nrf2 in pancreatic cancer [23]. Synnott et al. found that APR-246 exerted its anticancer activity partly by generating ROS and inducing the expression of SLC7A11-AS1 and xCT in breast cancer cells [27]. In addition, high expression of xCT has been found to contribute to chemoresistance in other cancers such as colorectal cancer [15], bladder cancer [11], lung cancer [28] and GC [29]. However, the role of SLC7A11-AS1 in GC chemoresistance remains unknown. Interestingly, we found that low SLC7A11-AS1 expression in GC was associated with poor progressionrelated survival, particularly PPS, but no significant association with OS was found in TCGA data. From OS analysis to stratified PPS analysis, low expression of SLC7A11-AS1 was shown to have a role in tumor progression, indicating that downregulated SLC7A11-AS1 may contribute to the chemoresistance that leads to tumor relapse and thus affect the survival and prognosis of patients. Additionally, high expression of XCT was significantly associated with GC survival but was more likely to shorten progression-related survival. Furthermore, we detected the expression of SLC7A11-AS1 and xCT in cisplatin-resistant patients and cells and found that SLC7A11-AS1 was downregulated and XCT was upregulated in cisplatin-resistant tissues and cells compared with cisplatin-sensitive tissues and cells. These results further supported that dysregulated SLC7A11-AS1 and XCT might contribute to chemoresistance. However, how do SLC7A11-AS1 and XCT regulate cisplatin resistance in GC?

In our previous study, we found that SLC7A11-AS1 was negatively correlated with xCT and that SLC7A11AS1 could suppress the expression of XCT in GC [22]. Consistent with our findings, Yuan et al. also reported that overexpression of SLC7A11-AS1 could significantly suppress the expression of XCT in ovarian cancer [26]. Moreover, Nafiseh et al. demonstrated that transcript isoform 6 of SLC7A11-AS1 significantly downregulated $\mathrm{XCT}$ by overlap between the complementary sequence region between exon 12 of $x C T$ and exons 2 and 3 of SLC7A11-AS1, which led to varicocele-related male infertility [30]. Here, we found that overexpression or depletion of SLC7A11-AS1 resulted in suppression or elevation of $x C T$ mRNA and protein levels in both cisplatin-sensitive and cisplatin-resistant cells. These results confirm that $x C T$ is a target gene regulated by SLC7A11-AS1, which can inhibit the expression of $x C T$. However, mounting evidence suggests that many types of cancer cells have increased levels of ROS compared with corresponding normal cells and that moderately high ROS levels are an important factor in promoting tumor progression [31, 32]. ROS are chemically reactive molecules that are involved in signaling cell growth and differentiation, regulating the activity of enzymes, mediating inflammation, and 
eliminating pathogens and foreign particles; ROS play essential roles in living organisms by regulating signal transduction [33]. A moderate increase in ROS can promote cell differentiation and proliferation $[34,35]$; conversely, excessive amounts of ROS can cause oxidative damage to DNA, proteins and lipids [36-38]. It is worth considering that if SLC7A11-AS1 always loses its suppressive effect on XCT, causing elevation of the intracellular GSH level, cancer cells will exhibit low levels of ROS that are insufficient for essential and normal function created by continual scavenging of ROS. Therefore, we speculated that there are other mechanisms regulating the SLC7A11-AS1/xCT axis to alleviate the continuous increase in intracellular $\mathrm{GSH}$, which maintains basic requirements for normal function. Through bioinformatic analysis, we found that SLC7A11-AS1 and xCT have binding sites for miR-33a-5p.

Further research found that miR-33a-5p was upregulated in cisplatin-resistant GC cells relative to sensitive GC cells and in SLC7A11-AS1-knockdown GC cells and that overexpression or inhibition of miR33a-5p remarkably reduced or enhanced the expression of SLC7A11-AS1 and xCT. In further work, we identified the effects of miR-33a-5p on SLC7A11-AS1 and xCT mediated by directly targeting the miR-33a$5 p$ binding sites. These results indicate that miR-33a-5p plays an important role in the maintenance of the dynamic intracellular antioxidation balance in cancer by negatively regulating the SLC7A11-AS1/xCT axis.

$\mathrm{xCT}$ is a target of SLC7A11-AS1, so we sought to determine whether SLC7A11-AS1 mediated cisplatin resistance through increasing production of GSH and decreasing production of ROS by regulating $\mathrm{XCT}$. Here, our results revealed that SLC7A11-AS1 in both cisplatin-resistant GC cells and cisplatin-sensitive GC cells could reduce intracellular GSH levels and increase intracellular ROS levels in vitro. Moreover, SLC7A11-AS1 reduced the intracellular level of sulfasalazine-induced GSH and enhanced the intracellular level of sulfasalazine-induced ROS in vitro. Moreover, we found that depletion of SLC7A11-AS1 reduced the intracellular level of sulfasalazine-induced ROS and enhanced the intracellular level of sulfasalazineinduced GSH. Our results indeed indicated that depleting SLC7A11-AS1 caused a significant accumulation of GSH by enhancing XCT expression in GC cells, leading to substantial ROS depletion. On the other hand, compared with cisplatin-sensitive GC cells, cisplatin-resistant GC cells showed higher levels of GSH and lower accumulation of ROS, suggesting that SLC7A11-AS1 expression is downregulated, which would promote the expression of $\mathrm{xCT}$, enhancing the transport of cystine into cells and leading to the synthesis of more GSH, which could effectively resist the high level of intracellular oxidative stress caused by ROS induced by chemotherapeutic drugs and thus produce drug resistance; this mechanism further supports the conclusion that the SLC7A11-AS1/XCT axis maintains the proper redox balance below the toxic threshold, inducing cisplatin resistance in cancer cells. The SGC7901/DDP xenograft model demonstrated the anticancer activity of SLC7A11-AS1, similar to that of the XCT inhibitor sulfasalazine, in vivo. The effects of SLC7A11-AS1 overexpression were like those of treatment with sulfasalazine, significantly potentiating cisplatin cytotoxicity in vivo. Therefore, our results clearly demonstrate that the SLC7A11-AS1/xCT axis is involved in GSH-mediated ROS elimination in the process of cisplatin resistance.

One of the cisplatin resistance mechanisms is the capacity of cancer cells to activate signaling pathways such as the mitogen-activated protein kinase (MAPK) pathway to provide additional survival and 
proliferative signals [39]. The p38MAPK-JNK signaling pathway is one of the canonical pathways, and activation of this signaling pathway plays a role in the maintenance of cisplatin resistance in squamous cell carcinoma [40], lung cancer [41] and ovarian cancer [42]. In GC cells, activation of the MAPK signaling pathway has been reported to be related to cisplatin resistance [43]. Apoptosis-signal regulating kinase 1 (ASK1), an upstream regulator of $\mathrm{JNK} / \mathrm{p} 38$, is an oxidative stress-responsive kinase that is activated by a variety of oxidative stress stimuli, including chemotherapeutic drugs, and has been shown to be induced by cisplatin [44]. However, whether SLC7A11-AS1 regulates the activation of ASK1-p38MAPK-JNK signaling related to cisplatin resistance requires further investigation in GC. Herein, consistent with previous studies, we confirmed that low expression of SLC7A11-AS1 activated ASK1-p38MAPK-JNK signaling by promoting XCT scavenging of ROS, which caused GC cisplatin resistance. The prosurvival or prodeath outcomes of p38/JNK activity are largely dependent on the duration of activation [45]. Shortterm activation, such as that occurring during cellular repair mechanisms, is protective, while sustained p38/JNK phosphorylation initiates apoptotic and necrotic cell death cascades [46-48]. In cisplatinresistant cells, cisplatin is rapidly metabolized, resulting in a transient increase in ROS by the SLC7A11AS1/xCT axis, which causes short-term activation of ASK1-p38MAPK-JNK signaling to initiate cellprotective mechanisms. These effects lead to resistance to cisplatin and cell survival in the presence of high concentrations of cisplatin. These findings strongly indicate that SLC7A11-AS1 and miR-33a-5p could potentially repress $\mathrm{xCT}$ and that miR-33a-5p suppresses SLC7A11-AS1 and $\mathrm{xCT}$ in turn, which results in the activation of ASK1-p38MAPK-JNK signaling, leading to GC cell growth and cisplatin resistance.

In addition to the above cisplatin resistance mechanism, a previous study reported that cisplatin metabolism was related to cisplatin import (CTR1) and export (ATP7A and ATP7B) and GSH biosynthesis (GCLC, GCLM, GSR, and 4F2HC) in the process of cisplatin resistance [11]. However, whether SLC7A11AS1 regulates cisplatin metabolism by mechanisms in addition to GSH biosynthesis by $\mathrm{xCT}$ is unknown. First, we compared the abovementioned genes between cisplatin-resistant and cisplatin-sensitive GC cells and found that the mRNA expression of the cisplatin export gene ATP7A and GSH biosynthesis-related genes GCLM and GSR was significantly upregulated in the resistant cell line SGC-7901/DDP. Then, we depleted SLC7A11-AS1 in GC cells and found that the cisplatin export gene ATP7A and GSH biosynthesisrelated genes GCLC and GCLM were significantly upregulated. These data indicated that low expression of SLC7A11-AS1 also induced cisplatin resistance by increasing the expression of the cisplatin export gene ATP7A and the GSH biosynthesis-related gene GCLM. Therefore, there are multiple ways in which SLC7A11-AS1 participates in the regulation of cisplatin resistance, mainly by up-regulating the expression of $\mathrm{XCT}$, promoting GSH synthesis to scavenge intracellular oxidative stress ROS produced by chemotherapeutic drugs, supplemented by increasing cisplatin export gene ATP7A and GSH biosynthesis gene GCLM to induce cisplatin resistance.

\section{Conclusion}

In summary, our work demonstrates that SLC7A11-AS1 suppresses cancer growth and reverses cisplatin chemoresistance by blocking $\mathrm{xCT}$ expression to dynamically balance intracellular oxidation through an 
interaction with miR-33a-5p. At the same time, the low expression of SLC7A11-AS1 also induces cisplatin resistance by increasing the cisplatin export gene ATP7A and the GSH biosynthesis gene GCLM. These findings suggest that the SLC7A11-AS1/xCT axis is a crucial therapeutic target to overcome platinumrelated resistance for $\mathrm{GC}$ treatment.

\section{Abbreviations}

GC: Gastric cancer; LncRNA: Long non-coding RNA; Quantitative real-time polymerase chain reaction: qRTPCR; RNA fluorescence in situ hybridization: RNA-FISH; Reactive oxygen species: ROS; Glutathione: GSH; Overall survival: OS; First progression: FP; Post-progression survival: PPS; Nuclear factor erythroid-2related factor 2: NRF2; Sulfasalazine: SSA.

\section{Declarations}

\section{Acknowledgments}

We thank Department of Gastrointestinal Surgery, The First Affiliated Hospital of Chengdu Medical College for providing the GC.

\section{Authors' contributions}

Y J Luo and J W Xiao designed the study. Y J Luo, W P Xiang, Z L Liu, L Yao, L H Tang ${ }^{1}$, W Tan ${ }^{1}$, P C Ye ${ }^{3}$, $\mathrm{J}$ W Xiao performed the in vitro and animal experiments. Y J Luo, W P Xiang, Z L Liu and J W Xiao analyzed the data and wrote the manuscript. All authors read and approved the final manuscript.

\section{Funding}

This study was supported by the National Natural Science Foundation of China Grant No. 81270561, and Sichuan Outstanding Youth Fund Project Grant NO. 2015JQ0060.

\section{Availability of data and materials}

All the data and materials supporting the conclusions were included in the main paper.

\section{Ethics approval and consent to participate}

This study was conducted in accordance with the Declaration of Helsinki principles. It was approved by the Ethics Committee of the First Affiliated Hospital of Chengdu Medical College, Chengdu, China. 


\section{Consent for publication}

All materials and images are original. No consent needs to declare.

\section{Competing interests}

The authors declare that they have no competing interests.

\section{Author details}

1 The Department of Gastrointestinal Surgery, The First Affiliated Hospital of Chengdu Medical College, Faculty of medicine, Chengdu, Sichuan, 610500 , People's Republic of China. ${ }^{2}$ The Department of Thoracic Surgery, The Affiliated Hospital of North Sichuan Medical College, Nanchong, Sichuan, 637000, People's Republic of China. ${ }^{3}$ The Department of Gastrointestinal Surgery, The Affiliated Hospital of North Sichuan Medical College, Nanchong, Sichuan, 637000, People's Republic of China. ${ }^{4}$ The Department of of Gastric Cancer Surgery, Tianjin Medical University Cancer Institute and Hospital, Tianjin, 300060, People's Republic of China

\section{References}

1. Bray F, et al. Global cancer statistics 2018: GLOBOCAN estimates of incidence and mortality worldwide for 36 cancers in 185 countries. CA Cancer J Clin. 2018;68(6):394-424.

2. Ferlay $\mathrm{J}$, et al. Cancer incidence and mortality worldwide: sources, methods and major patterns in GLOBOCAN 2012. Int J Cancer. 2015;136(5):E359-86.

3. Chen W, et al. Cancer statistics in China, 2015. CA Cancer J Clin. 2016;66(2):115-32.

4. Lutz MP, et al. Highlights of the EORTC St. Gallen International Expert Consensus on the primary therapy of gastric, gastroesophageal and oesophageal cancer - differential treatment strategies for subtypes of early gastroesophageal cancer. Eur J Cancer. 2012;48(16):2941-53.

5. Cunningham $D$, et al. Perioperative chemotherapy versus surgery alone for resectable gastroesophageal cancer. N Engl J Med. 2006;355(1):11-20.

6. Sasako $M$, et al. Five-year outcomes of a randomized phase III trial comparing adjuvant chemotherapy with S-1 versus surgery alone in stage II or III gastric cancer. J Clin Oncol. 2011;29(33):4387-93.

7. Kang YK, et al. S-1 plus leucovorin and oxaliplatin versus S-1 plus cisplatin as first-line therapy in patients with advanced gastric cancer (SOLAR): a randomised, open-label, phase 3 trial. Lancet Oncol. 2020;21(8):1045-56.

8. Koizumi W, et al. Impacts of fluorouracil-metabolizing enzymes on the outcomes of patients treated with S-1 alone or S-1 plus cisplatin for first-line treatment of advanced gastric cancer. Int J Cancer. 
2010;126(1):162-70.

9. Galluzzi L, et al. Systems biology of cisplatin resistance: past, present and future. Cell Death Dis. 2014;5:e1257.

10. Galluzzi L, et al. Molecular mechanisms of cisplatin resistance. Oncogene. 2012;31(15):1869-83.

11. Drayton RM, et al. Reduced expression of miRNA-27a modulates cisplatin resistance in bladder cancer by targeting the cystine/glutamate exchanger SLC7A11. Clin Cancer Res. 2014;20(7):19902000.

12. Russo A, et al. Selective modulation of glutathione levels in human normal versus tumor cells and subsequent differential response to chemotherapy drugs. Cancer Res. 1986;46(6):2845-8.

13. Wu G, et al. Glutathione metabolism and its implications for health. J Nutr. 2004;134(3):489-92.

14. Sato $\mathrm{H}$, et al. Cloning and expression of a plasma membrane cystine/glutamate exchange transporter composed of two distinct proteins. J Biol Chem. 1999;274(17):11455-8.

15. Ma MZ, et al. Xc- inhibitor sulfasalazine sensitizes colorectal cancer to cisplatin by a GSH-dependent mechanism. Cancer Lett. 2015;368(1):88-96.

16. Drayton RM, et al. Reduced expression of miRNA-27a modulates cisplatin resistance in bladder cancer by targeting the cystine/glutamate exchanger SLC7A11. Clin Cancer Res. 2014;20(7):19902000.

17. Wang SF, et al. Mitochondrial dysfunction enhances cisplatin resistance in human gastric cancer cells via the ROS-activated GCN2-elF2alpha-ATF4-xCT pathway. Oncotarget. 2016;7(45):74132-51.

18. Luo YJ, et al. Non-coding RNA in drug resistance of gastric cancer. World J Gastrointest Oncol. 2019;11(11):957-70.

19. Wei L, et al. Noncoding RNAs in gastric cancer: implications for drug resistance. Mol Cancer. 2020;19(1):62.

20. Cheng $\mathrm{C}$, et al., Knockdown of long non-coding RNA HOTAIR inhibits cisplatin resistance of gastric cancer cells through inhibiting the PI3K/Akt and Wnt/beta-catenin signaling pathways by upregulating miR-34a. Int J Biol Macromol, 2018. 107(Pt B): p. 2620-2629.

21. YiRen $\mathrm{H}$, et al. Long noncoding RNA MALAT1 regulates autophagy associated chemoresistance via miR-23b-3p sequestration in gastric cancer. Mol Cancer. 2017;16(1):174.

22. Luo Y, et al. Decreased expression of the long non-coding RNA SLC7A11-AS1 predicts poor prognosis and promotes tumor growth in gastric cancer. Oncotarget. 2017;8(68):112530-49.

23. Yang Q, et al. IncRNA SLC7A11-AS1 Promotes Chemoresistance by Blocking SCF(beta-TRCP)Mediated Degradation of NRF2 in Pancreatic Cancer. Mol Ther Nucleic Acids. 2020;19:974-85.

24. Liu Y, et al. LncRNA SLC7A11-AS1 Contributes to Lung Cancer Progression Through Facilitating TRAIP Expression by Inhibiting miR-4775. Onco Targets Ther. 2020;13:6295-302.

25. Liang J, et al. Comprehensive analysis of TGF-beta-induced mRNAs and ncRNAs in hepatocellular carcinoma. Aging. 2020;12(19):19399-420. 
26. Yuan J, Liu Z, Song R. Antisense IncRNA As-SLC7A11 suppresses epithelial ovarian cancer progression mainly by targeting SLC7A11. Pharmazie. 2017;72(7):402-7.

27. Synnott NC, et al. The Mutant p53-Targeting Compound APR-246 Induces ROS-Modulating Genes in Breast Cancer Cells. Transl Oncol. 2018;11(6):1343-9.

28. Horibe $S$, et al. CD44v-dependent upregulation of $x C T$ is involved in the acquisition of cisplatinresistance in human lung cancer A549cells. Biochem Biophys Res Commun. 2018;507(1-4):426-32.

29. Wang SF, et al., Activated Integrated Stress Response Induced by Salubrinal Promotes Cisplatin Resistance in Human Gastric Cancer Cells via Enhanced xCT Expression and Glutathione Biosynthesis. Int J Mol Sci, 2018. 19(11).

30. Sanei-Ataabadi N, Mowla SJ, Nasr-Esfahani MH. Transcript Isoforms of SLC7A11-AS1 Are Associated With Varicocele-Related Male Infertility. Front Genet. 2020;11:1015.

31. Szatrowski TP, Nathan CF. Production of large amounts of hydrogen peroxide by human tumor cells. Cancer Res. 1991;51(3):794-8.

32. Huang JS, et al. Oxidative stress enhances Axl-mediated cell migration through an Akt1/Rac1dependent mechanism. Free Radic Biol Med. 2013;65:1246-56.

33. Trachootham D, Alexandre J, Huang P. Targeting cancer cells by ROS-mediated mechanisms: a radical therapeutic approach? Nat Rev Drug Discov. 2009;8(7):579-91.

34. Boonstra J, Post JA. Molecular events associated with reactive oxygen species and cell cycle progression in mammalian cells. Gene. 2004;337:1-13.

35. Schafer FQ, Buettner GR. Redox environment of the cell as viewed through the redox state of the glutathione disulfide/glutathione couple. Free Radic Biol Med. 2001;30(11):1191-212.

36. Radak Z, et al. Age-associated neurodegeneration and oxidative damage to lipids, proteins and DNA. Mol Aspects Med. 2011;32(4-6):305-15.

37. Cobbaut M, Van Lint J, Function and Regulation of Protein Kinase D in Oxidative Stress: A Tale of Isoforms. Oxid Med Cell Longev, 2018. 2018: p. 2138502.

38. Cui S, et al. Folic acid modulates VP01 DNA methylation levels and alleviates oxidative stressinduced apoptosis in vivo and in vitro. Redox Biol. 2018;19:81-91.

39. Benhar M, Engelberg D, Levitzki A. Cisplatin-induced activation of the EGF receptor. Oncogene. 2002;21(57):8723-31.

40. Kong LR, et al. MEK Inhibition Overcomes Cisplatin Resistance Conferred by SOS/MAPK Pathway Activation in Squamous Cell Carcinoma. Mol Cancer Ther. 2015;14(7):1750-60.

41. Cai Y, Dong ZY, Wang JY. LncRNA NNT-AS1 is a major mediator of cisplatin chemoresistance in nonsmall cell lung cancer through MAPK/Slug pathway. Eur Rev Med Pharmacol Sci. 2018;22(15):4879-87.

42. Liang $F$, et al. The crosstalk between STAT3 and p53/RAS signaling controls cancer cell metastasis and cisplatin resistance via the Slug/MAPK/PI3K/AKT-mediated regulation of EMT and autophagy. Oncogenesis. 2019;8(10):59. 
43. Zhou J, Chen Q. Poor expression of microRNA-135b results in the inhibition of cisplatin resistance and proliferation and induces the apoptosis of gastric cancer cells through MST1-mediated MAPK signaling pathway. FASEB J. 2019;33(3):3420-36.

44. Yuan ZQ, et al. AKT2 inhibition of cisplatin-induced JNK/p38 and Bax activation by phosphorylation of ASK1. IMPLICATION OF AKT2 IN CHEMORESISTANCE. J Biol Chem. 2016;291(43):22847.

45. Ogier JM, Nayagam BA, Lockhart PJ. ASK1 inhibition: a therapeutic strategy with multi-system benefits. J Mol Med (Berl). 2020;98(3):335-48.

46. Tomida T, Takekawa M, Saito H. Oscillation of p38 activity controls efficient pro-inflammatory gene expression. Nat Commun. 2015;6:8350.

47. Liu H, Lo CR, Czaja MJ. NF-kappaB inhibition sensitizes hepatocytes to TNF-induced apoptosis through a sustained activation of JNK and c-Jun. Hepatology. 2002;35(4):772-8.

48. Shinohara $M$, et al. Silencing glycogen synthase kinase-3beta inhibits acetaminophen hepatotoxicity and attenuates JNK activation and loss of glutamate cysteine ligase and myeloid cell leukemia sequence 1. J Biol Chem. 2010;285(11):8244-55.

\section{Figures}

A

B
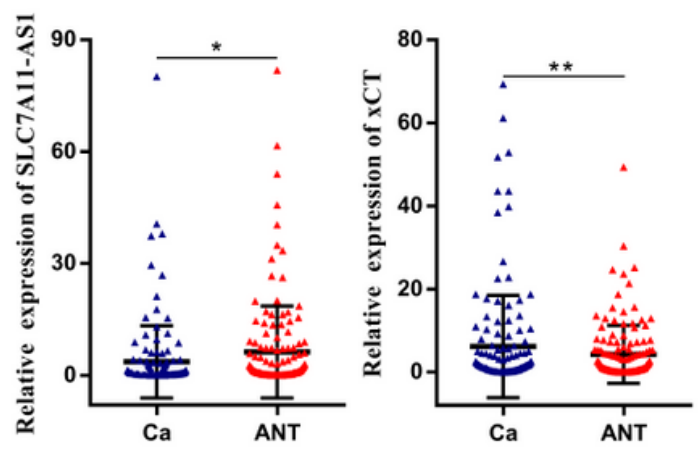

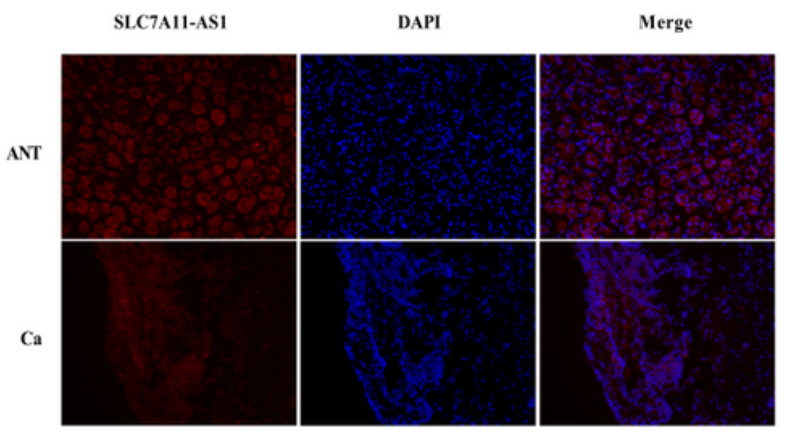

ANT

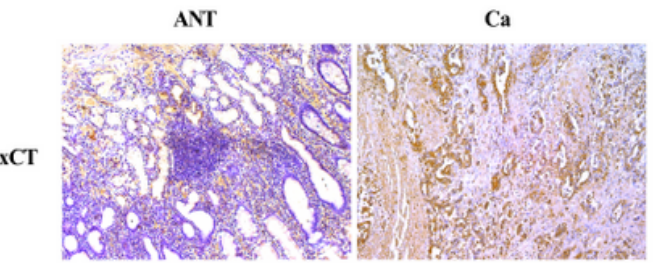

C

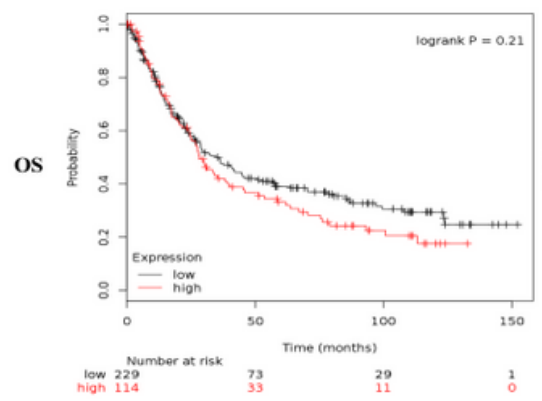

SLC7A11-AS1
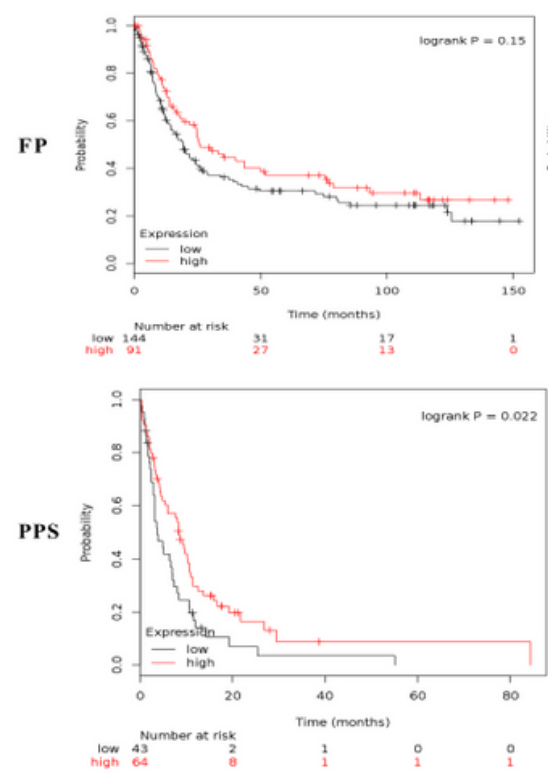

xCT
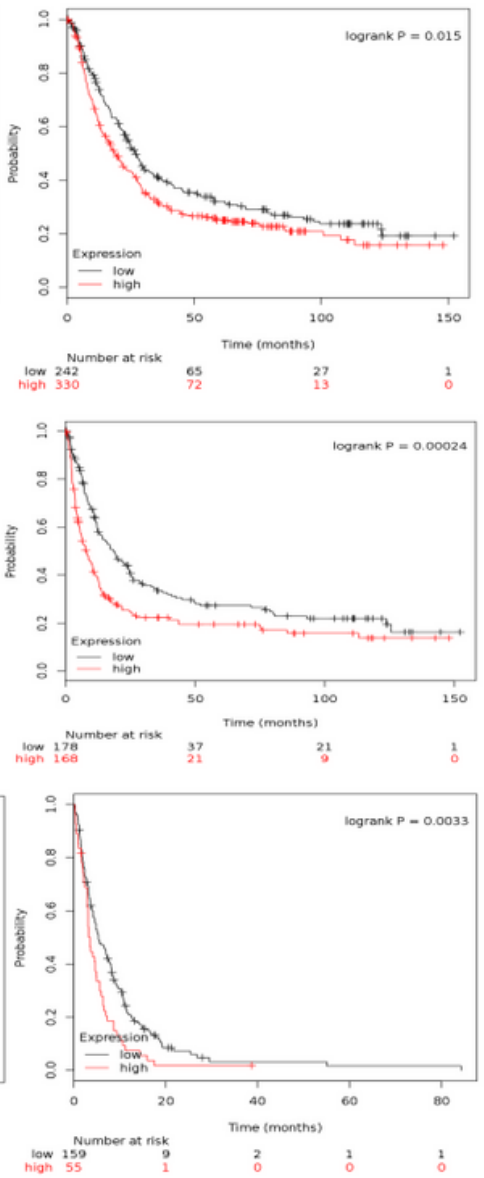
Figure 1

SLC7A11-AS1 and xCT expression levels and prognosis were assessed in human GC patients. (A) SLC7A11-AS1 and XCT expression levels were assessed in human GC tissues $(C a, n=140)$ and paired adjacent noncancerous tissues (ANT, $n=140$ ). Expression levels were normalized to $\beta$-actin levels. Results are shown as mean \pm SD. (B) Fluorescence in situ hybridization (FISH) for SLC7A11-AS1 and immunohistochemical staining for XCT in pairs of representative gastric cancer tissues (Ca) with adjacent non-tumorous tissues (ANT) (200x). (C) Kaplan-Meier analysis of OS (Overall Survival), FP ( First Progression) and PPS (Post Progression Survival) for expression of SLC7A11-AS1 ( $n=343)$ and $x C T$ $(n=572)$ in human GC patients. $\left({ }^{*} P<0.05,{ }^{*} P<0.01\right)$.

A

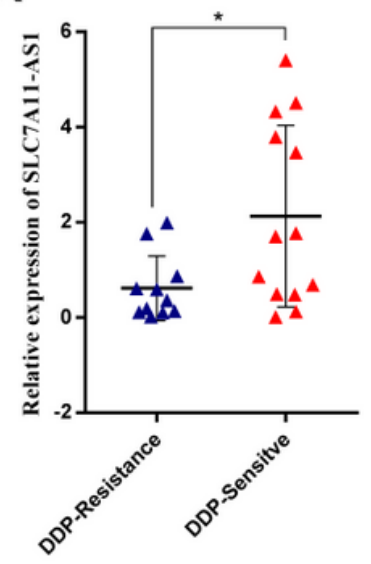

C

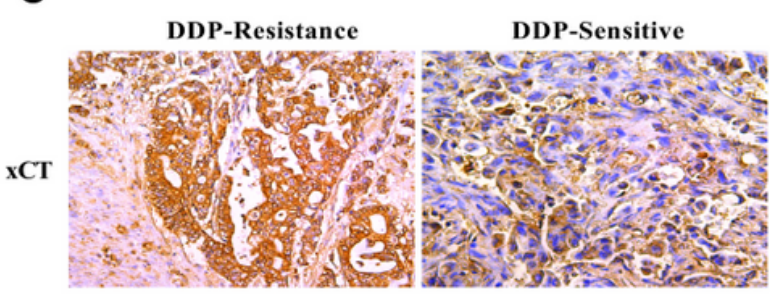

E
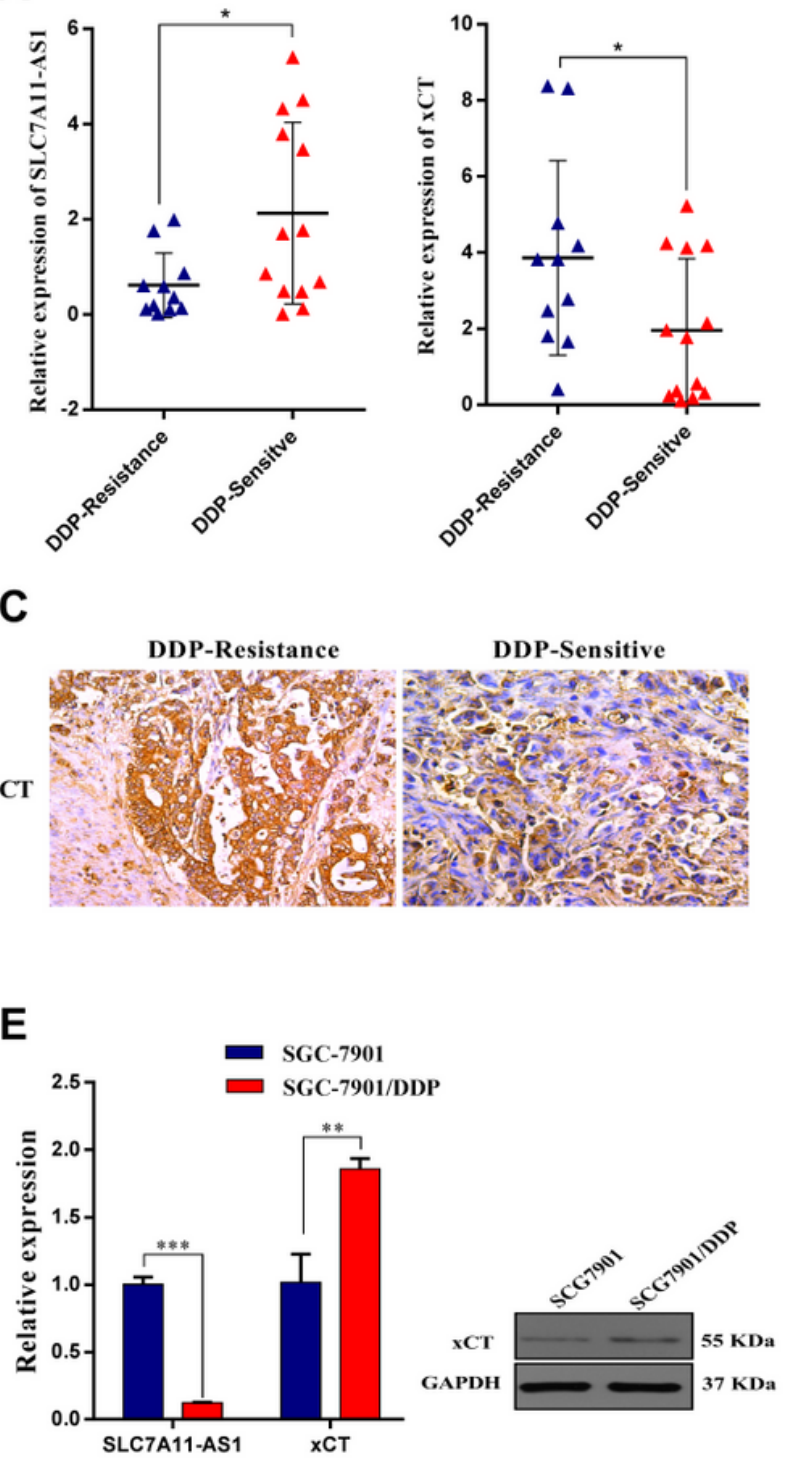

B

D

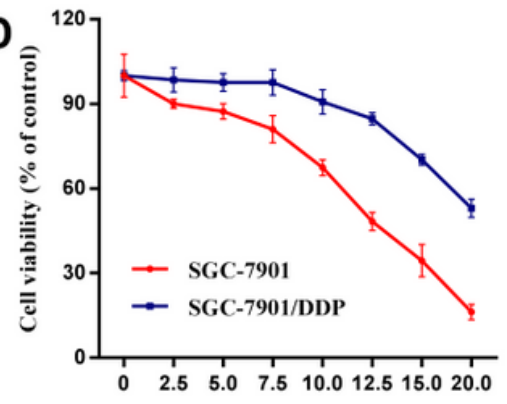

F

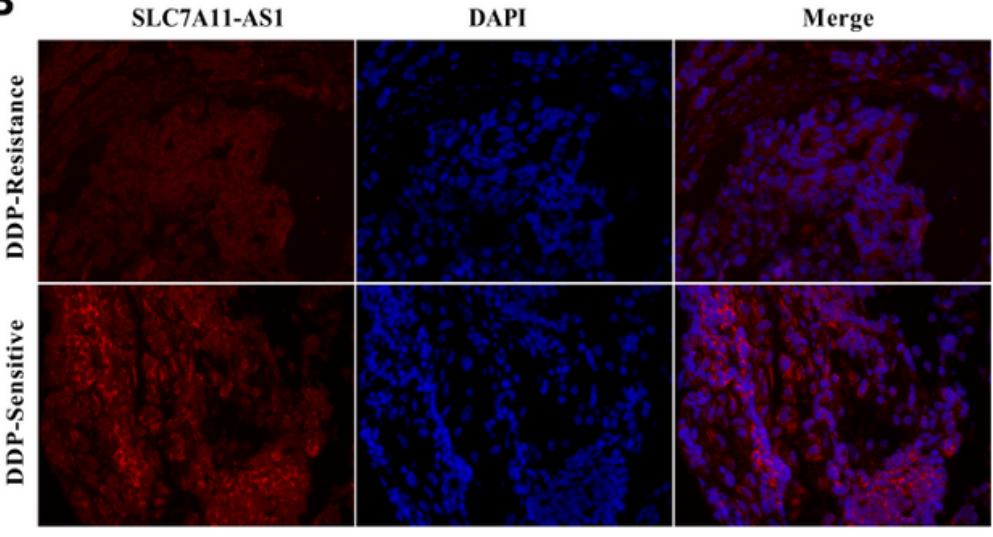

Cisplatin $(\mu \mathrm{M})$

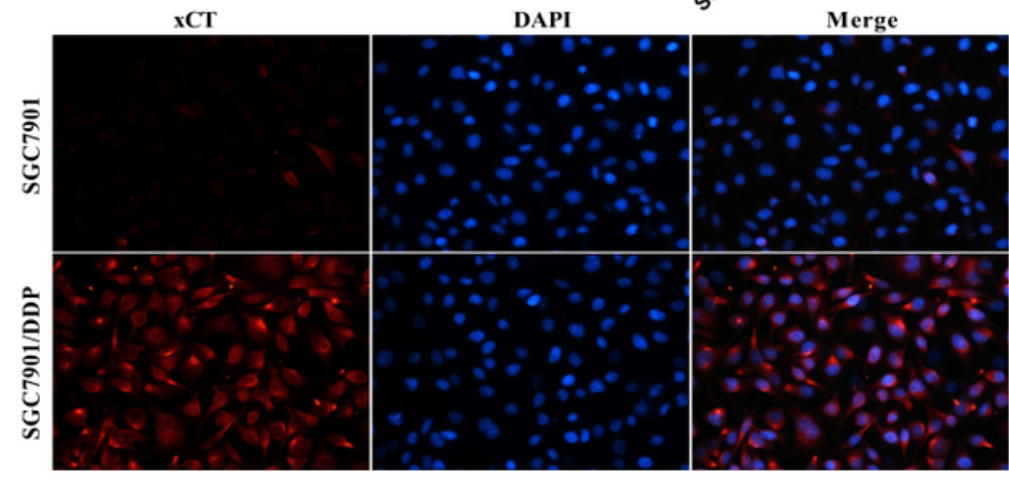

Figure 2 
Association of SLC7A11-AS1 and XCT expression with chemotherapy outcome in the patients with GC. (A) SLC7A11-AS1 and $x C T$ expression in cisplatin-sensitive $(n=13)$ and -resistant $(n=11)$ tumorous tissues. Expression levels were normalized to $\beta$-actin levels. Results are shown as mean \pm SD. (B) Fluorescence in situ hybridization (FISH) for SLC7A11-AS1 in cisplatin-sensitive and -resistant tumorous tissues (200x). (C) Immunohistochemical staining for XCT in cisplatin-sensitive and -resistant tumorous tissues (400x). (D) Characterization of cisplatin-sensitive and -resistant SGC-7901 cell lines. Cell viability was assessed to show cisplatin sensitivity in cisplatin sensitive SGC-7901 cells and their resistant SGC$7901 /$ DDP cells. Error bars indicate SD, $n=4$. Half inhibitor effective concentrations (IC50) to cisplatin in these SGC-7901 cells and SGC-7901/DDP cells. Results are shown as mean \pm SD. (E) SLC7A11-AS1 and xCT expression in cisplatin sensitive SGC-7901 cells and their resistant SGC-7901/DDP cells. (F) Immunofluorescence staining for $X C T$ in SGC-7901 and SGC-7901/DDP cells (200x). $\left({ }^{\star} P<0.05,{ }^{\star \star \star} P<0.01\right.$, $\star * \star P<0.001$, two-tailed Student's t-test). 
A
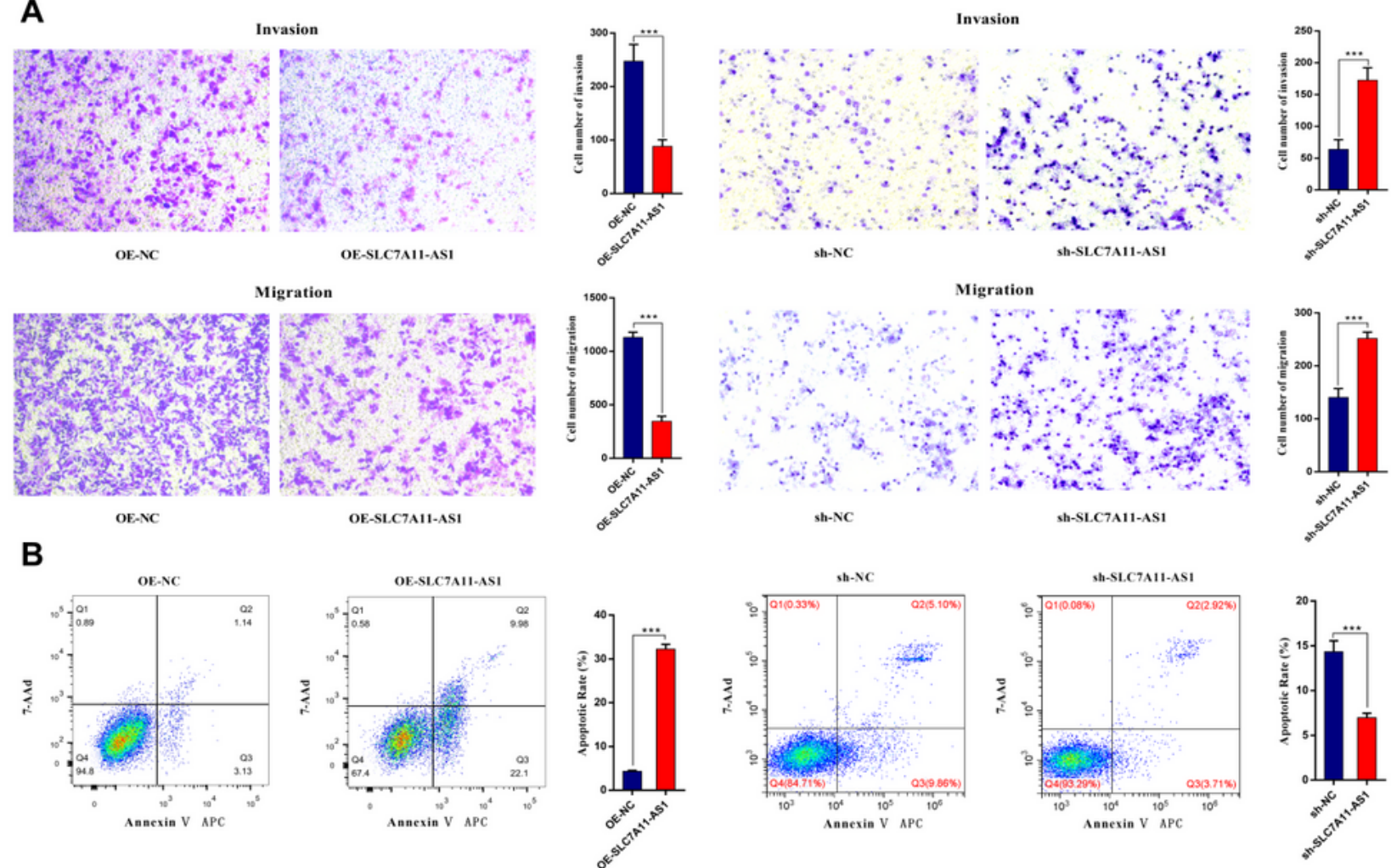

C

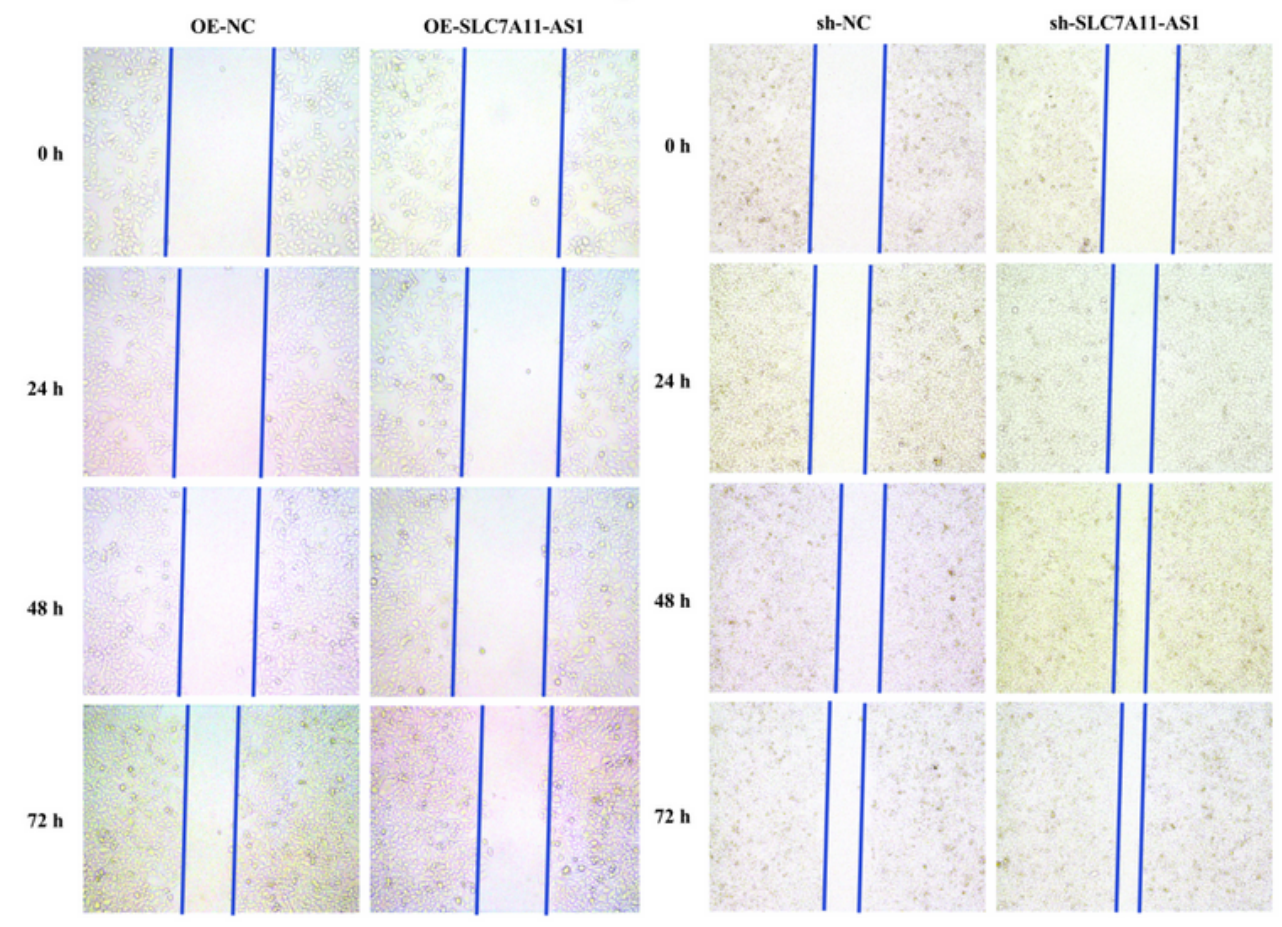

Figure 3

The function of SLC7A11-AS1 on human gastric cancer cells. (A) The cell invasive and migratory ability of SGC7901 cells were determined by cell transwell migration and invasion assays after up-regulating or down-regulating SLC7A11-AS1. (B) Wound-healing assay was performed to analyze the effect of SLC7A11-AS1 on cell migration. (C) The function of SLC7A11-AS1 on cell apotosis was measured by 
flowcytometry after PI and APC staining in SGC7901. Error bars suggest mean \pm SD. $\left({ }^{*} P<0.05,{ }^{*} P<0.01\right.$, $\star \star \star P<0.001$, two-tailed Student's t-test).
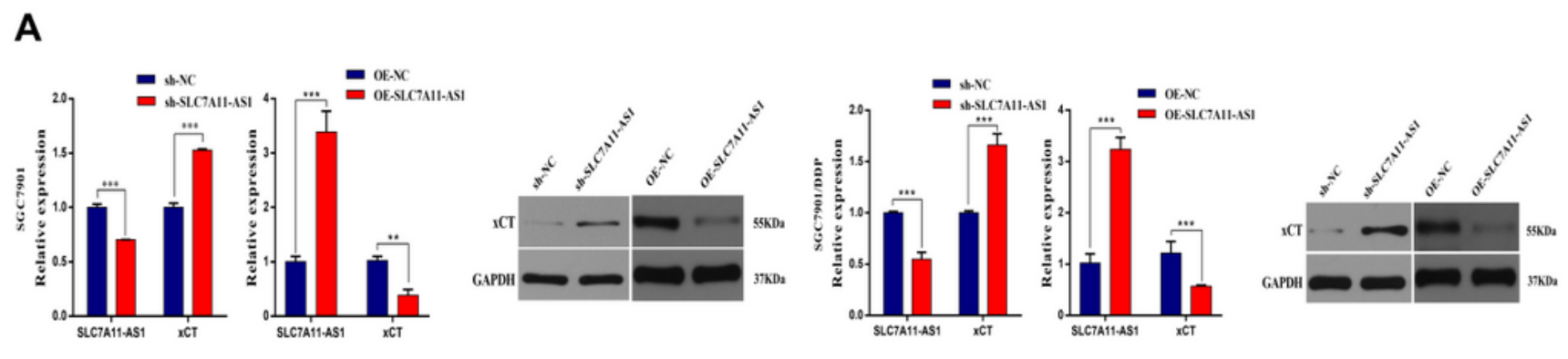

B
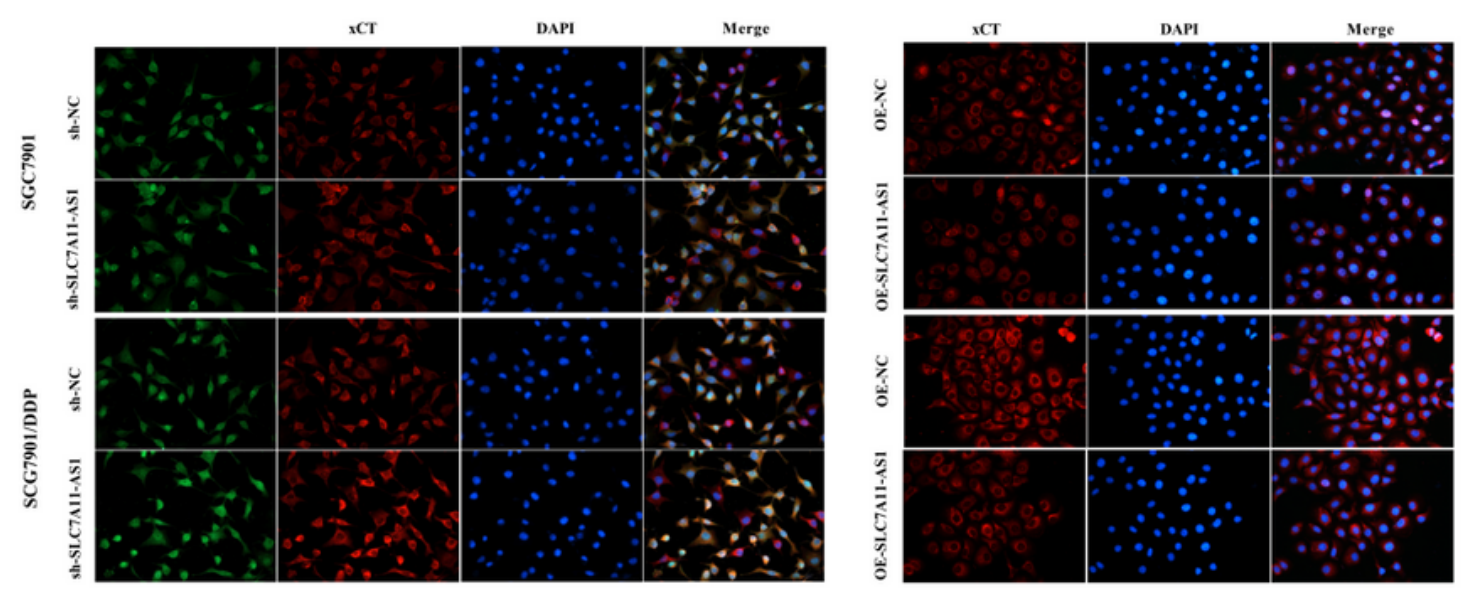

C

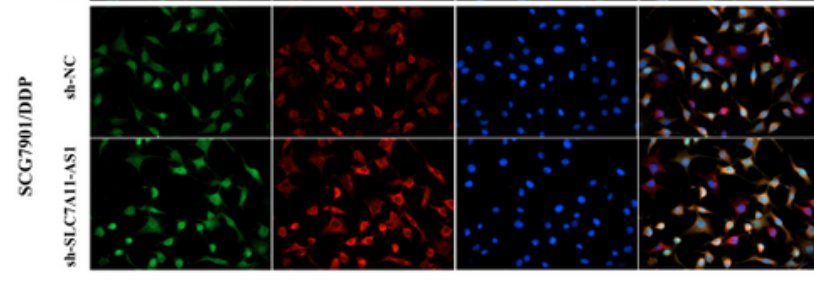

E
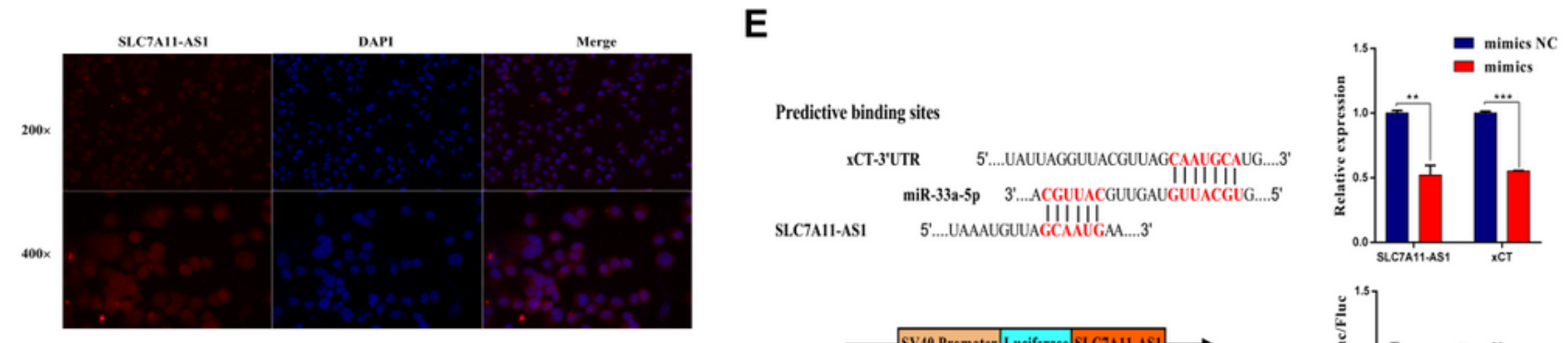

D
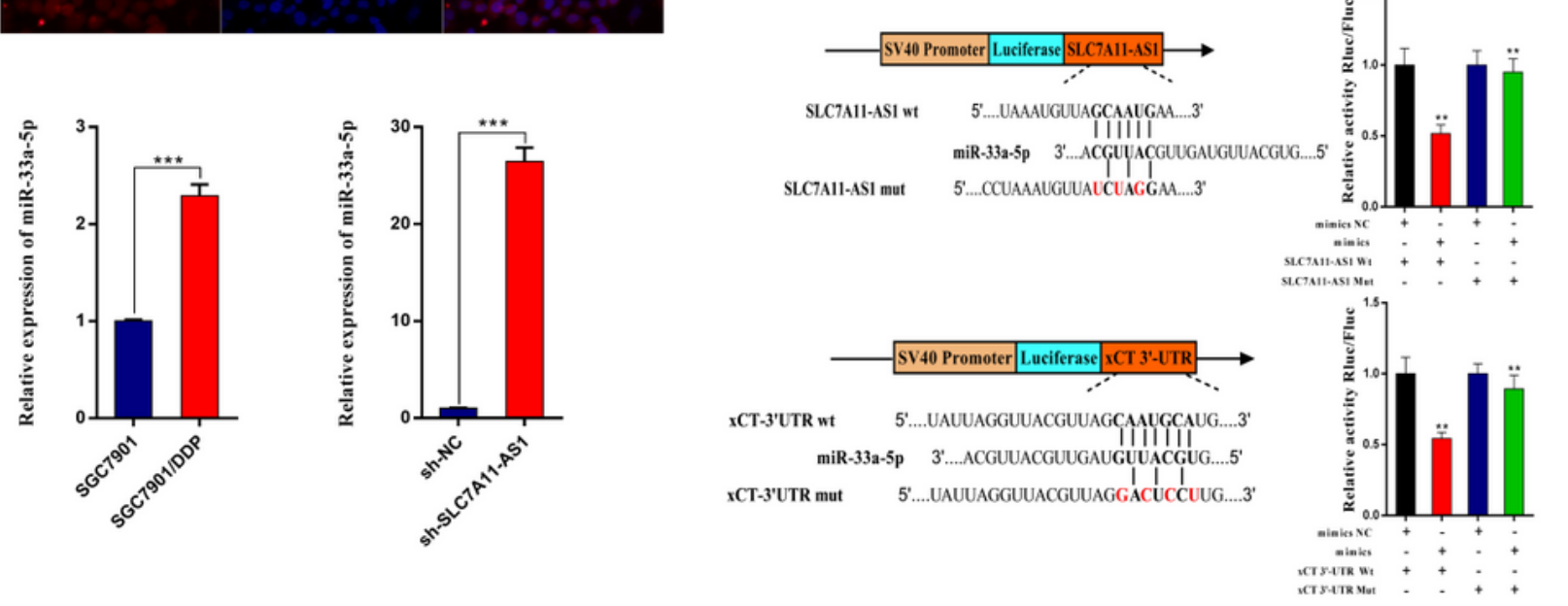

Figure 4

SLC7A11-AS1 suppress $x C T$ expression and miR-33a-5p directly targeting SLC7A11-AS1 and xCT. (A) The level of SLC7A11-AS1 and XCT expression in cisplatin sensitive SGC-7901 cells and their resistant SGC7901/DDP cells after up-regulating or down-regulating SLC7A11-AS1. (B) The immunofluorescence 
staining of xCT (red), SLC7A11-AS1 vector (green), and DAPI (blue) in SGC-7901 and SGC-7901/DDP cells. (C) Fluorescence in situ hybridization (FISH) for SLC7A11-AS1 in SGC-7901 cells. (D) The level of miR-33a-5p expression in SGC-7901 and SGC-7901/DDP cells and in SGC-7901 cells after downregulating SLC7A11-AS1. (E) SLC7A11-AS1 and xCT directly bound to miR-33a-5p. Error bars suggest mean \pm SD. $\left({ }^{* * P}<0.01,{ }^{* * * P}<0.001\right.$, two-tailed Student's t-test).
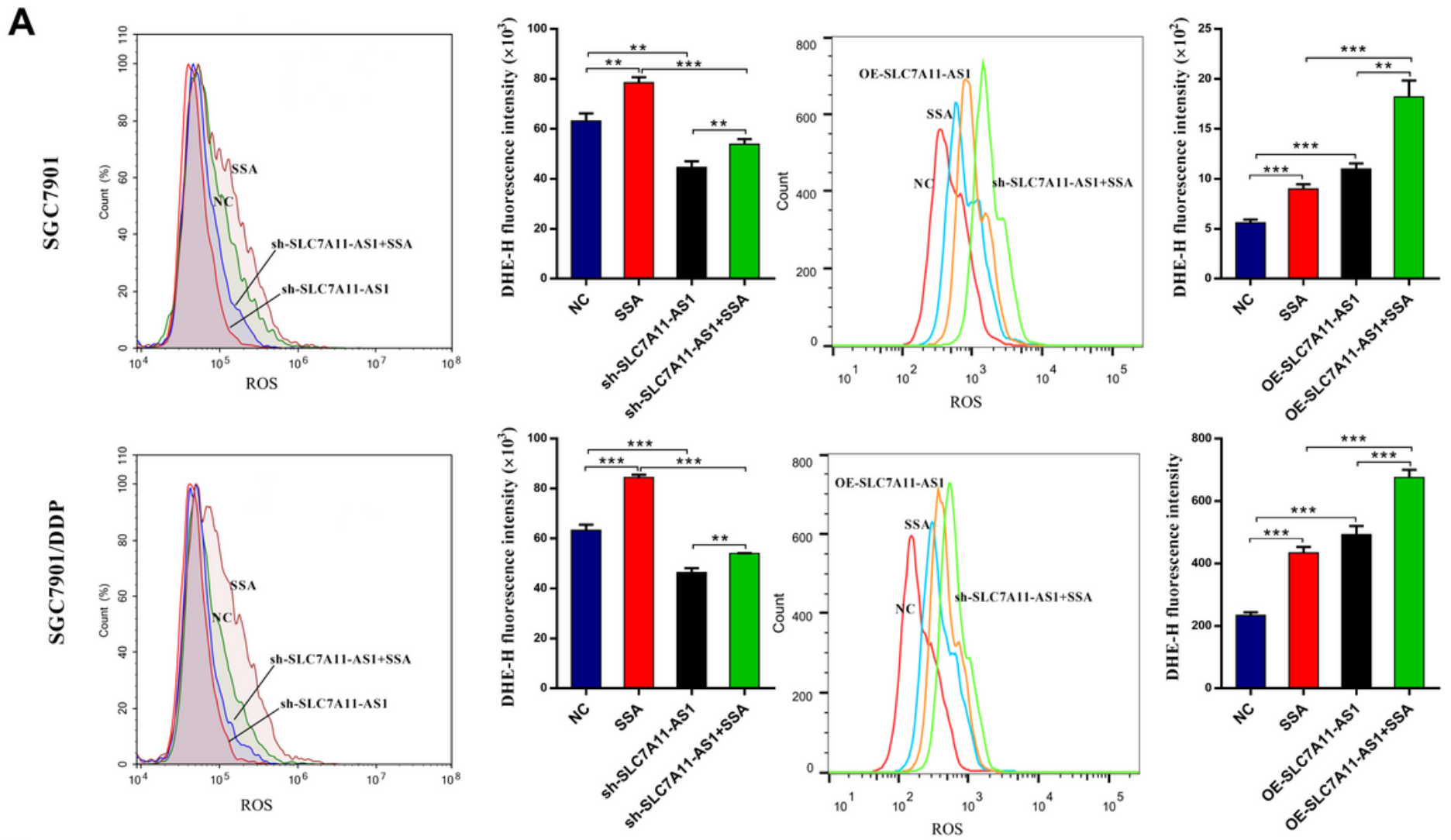

B

SCG7901
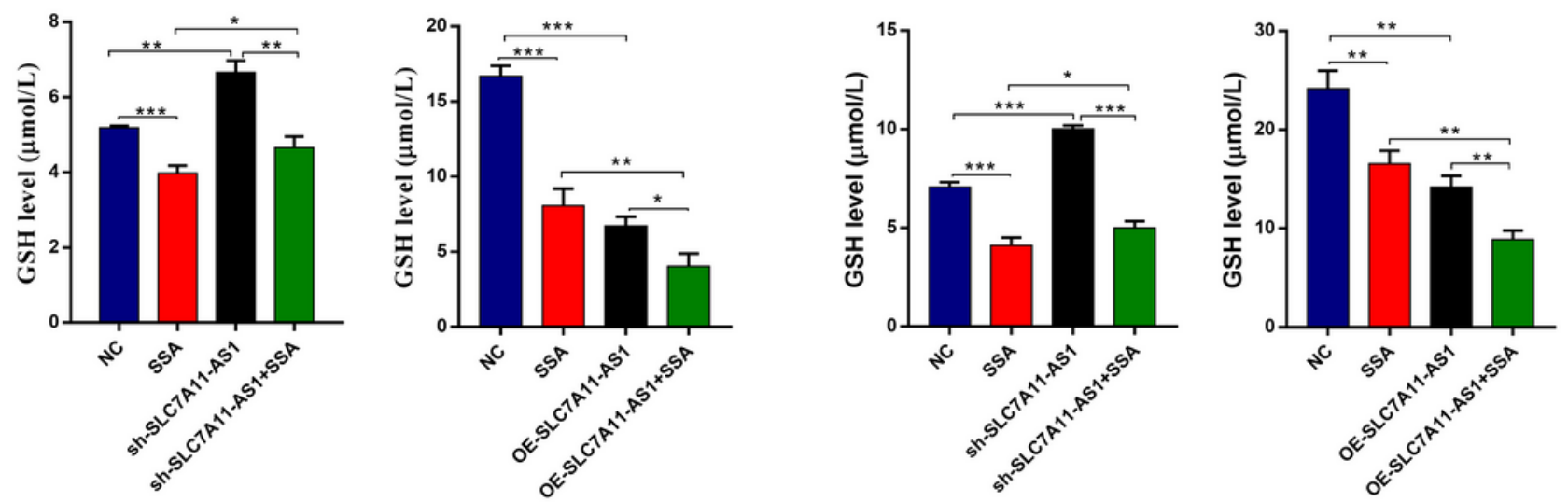

\section{Figure 5}

SLC7A11-AS1 regulated the intracellular ROS and GSH. (A) The level of intracellular ROS in cisplatin sensitive SGC-7901 cells and their resistant SGC-7901/DDP cells after up-regulating or down-regulating SLC7A11-AS1 and treatment with xCT inhibitor, Sulfasalazine (SSA). (B) The level of intracellular GSH in SGC-7901 and SGC-7901/DDP cells after up-regulating or down-regulating SLC7A11-AS1 and treatment 
with $x C T$ inhibitor, SSA. Error bars suggest mean \pm SD. $\left({ }^{\star} P<0.05,{ }^{\star} P P<0.01,{ }^{\star \star *} P<0.001\right.$, two-tailed Student's t-test).

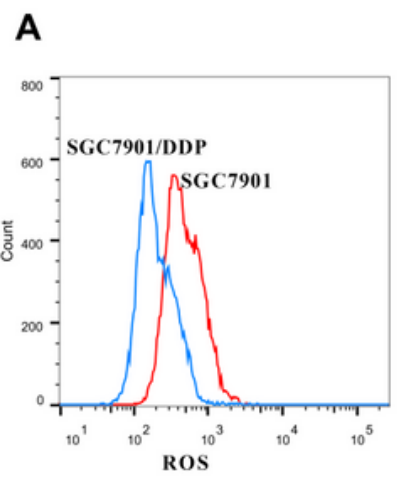

c
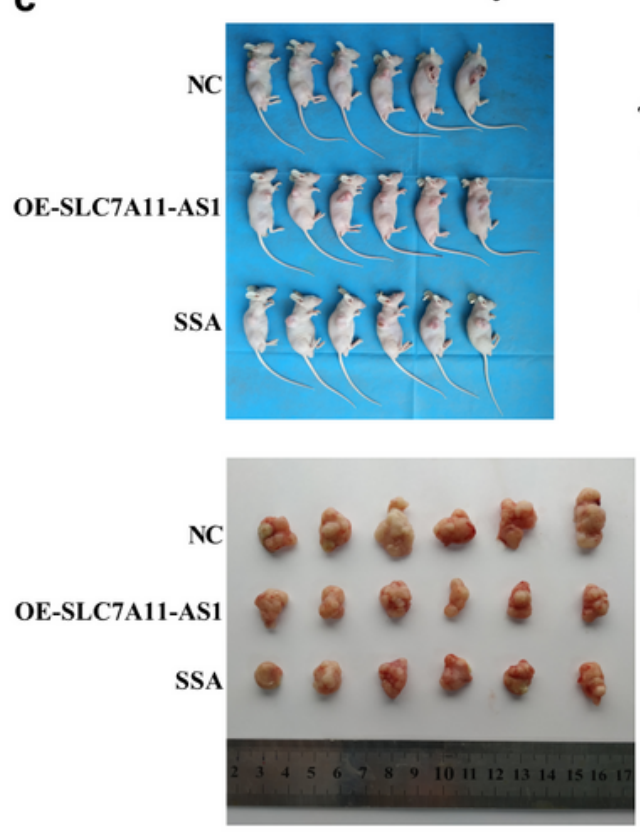

B
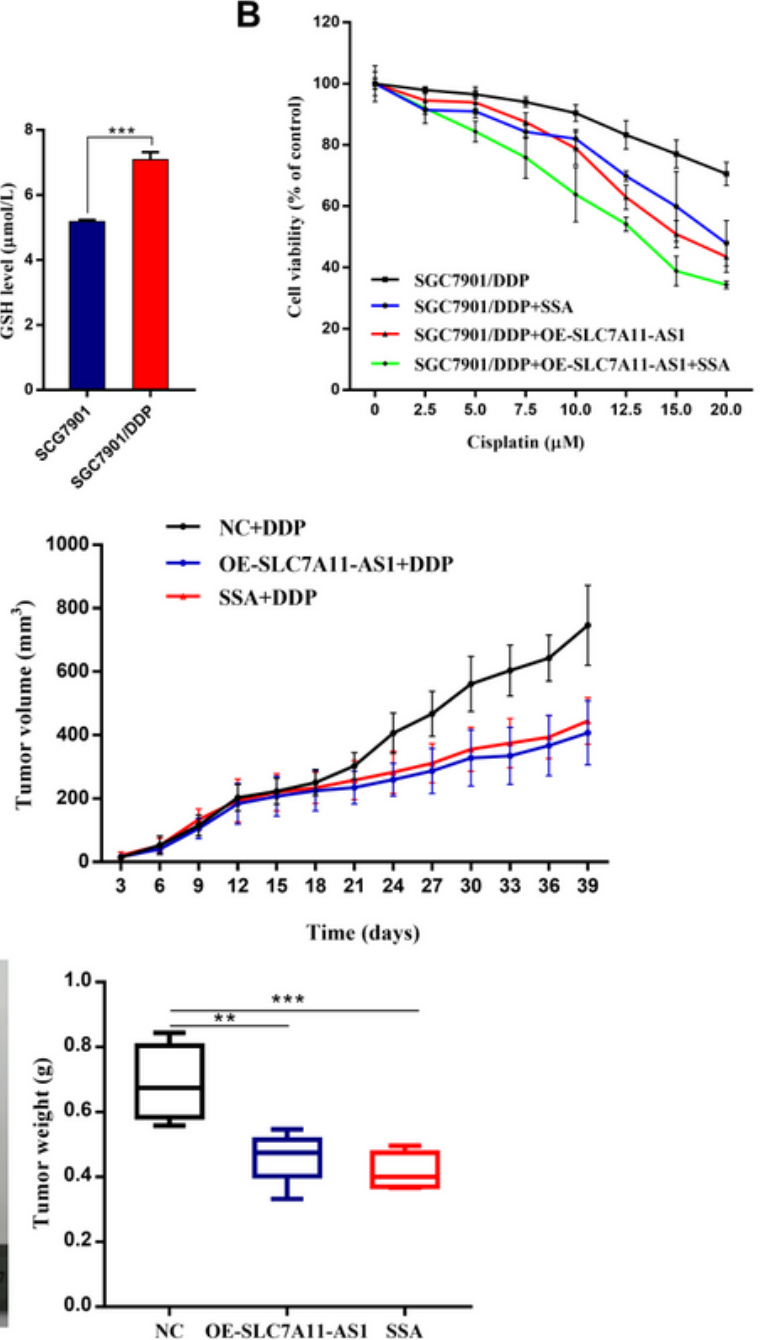

D

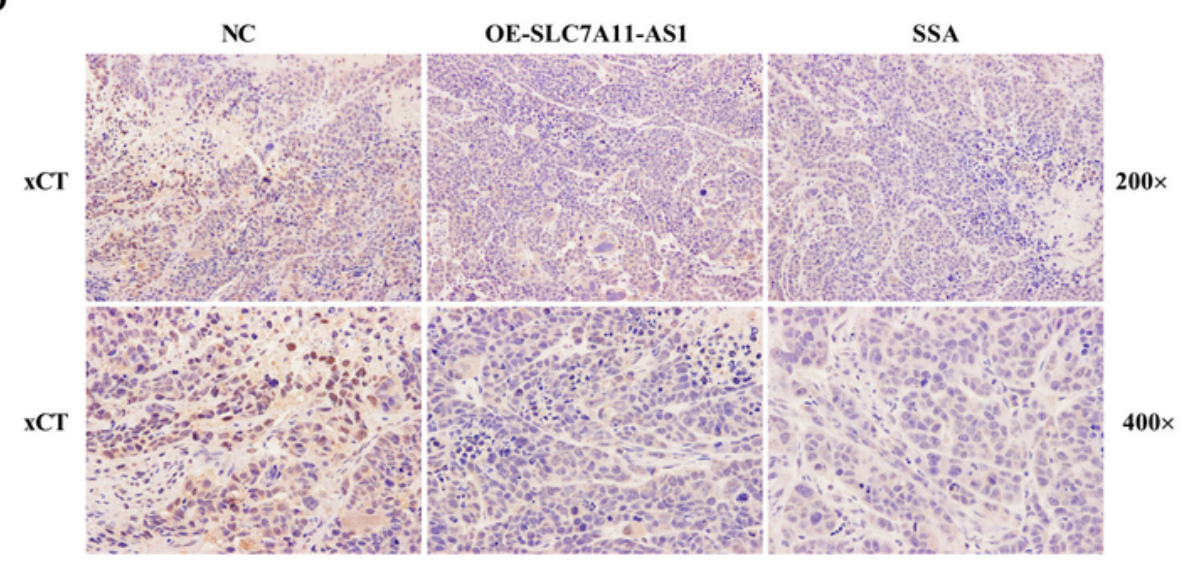

Figure 6

SLC7A11-AS1/xCT axis mediated cisplatin resistance. (A) The level of intracellular ROS and GSH in cisplatin sensitive SGC-7901 cells and their resistant SGC-7901/DDP cells. (B) Cell viability was assessed to show cisplatin sensitivity in cisplatin resistant SGC-7901/DDP cells after up-regulating SLC7A11-AS1 
or treatment with xCT inhibitor, Sulfasalazine (SSA). (C) Representative images of mice bearing SGC7901/DDP tumors from different treatment groups and the tumor volume growth curves and weight curves over the study period after injections of different treatment groups $(n=6)$. (D) Immunohistochemical staining for XCT in different xenograft tumor tissues on the 39th day after injections. Error bars suggest mean \pm SD. ( ${ }^{*} P<0.05$, ${ }^{*} \mathrm{P}<0.01$, ${ }^{*} * \mathrm{P}<0.001$, two-tailed Student's t-test).

\section{A}
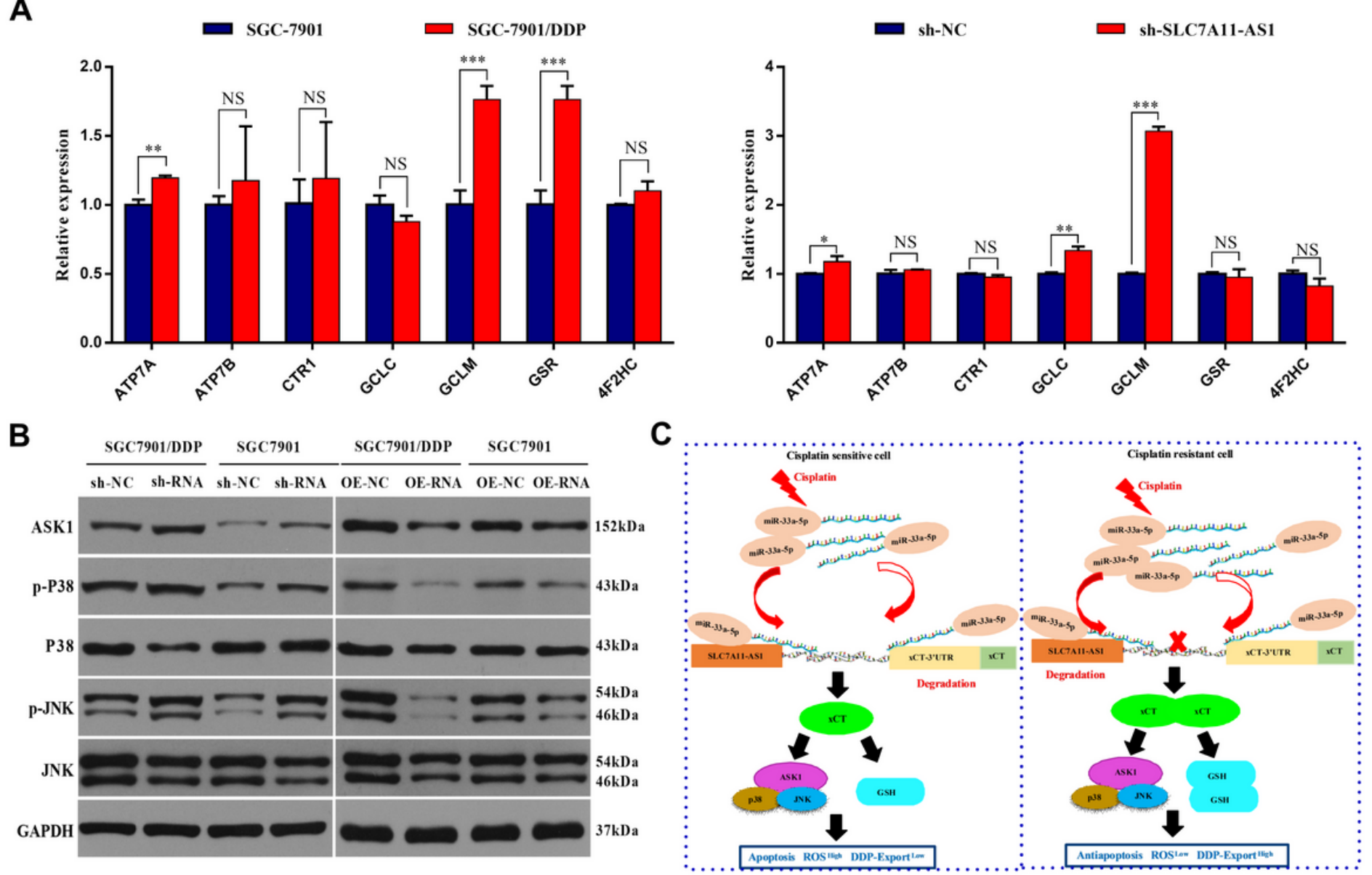

\section{Figure 7}

Analysis of candidate pathways mediating cisplatin resistance in sensitive and resistant cells. (A) change in expression of three mRNAs concerned with cisplatin import (CTR1), export (ATP7A, ATP7B) and GSH biosynthesis (GCLC, GCLM, GSR, 4F2HC) in cisplatin-sensitive (SGC7901) and -resistant (SGC7901/DDP) cells. (B) The level of p38MAPK-JNK signaling molecules in cisplatin sensitive SGC-7901 cells and their resistant SGC-7901/DDP cells after up-regulating or down-regulating SLC7A11-AS1. (C) A proposed working diagram of SLC7A11-AS1/xCT axis in regulating of cisplatin resistance. SLC7A11-AS1 suppresses XCT in transcriptional level by interacting with miR-33a-5p to lower ROS for the maintenance cisplatin resistance. Error bars suggest mean $\pm S D$. $\left({ }^{\star} P<0.05,{ }^{\star} * P<0.01,{ }^{\star} * \star P<0.001\right.$, two-tailed Student's $t$ test).

\section{Supplementary Files}


This is a list of supplementary files associated with this preprint. Click to download.

- Additionalfiles.doc 